\title{
A Cross-Linking Methodology for Fully Atomistic Models of Hydroxyl-Terminated Polybutadiene and Determination of Mechanical Properties
}

\author{
Supporting Information \\ Garrett M. Tow and Edward J. Maginn \\ Department of Chemical and Biomolecular Engineering, University of Notre Dame, Notre Dame, \\ Indiana 46556, United States \\ E-mail: ed@nd.edu
}




\section{Cross-Linking Minimization Details}

To ensure the desired stereochemistry, the following dihedral types, shown in Table S4, were modified during cross-linking minimization: $1,2,13,14,26,57,58,91,92,93,94,95,96$. These dihedrals were changed to have $\mathrm{K}_{2-4}$ all equal to 0.0 and $\mathrm{K}_{1}$ equal to +28.00 or $-28.00 \mathrm{kcal} / \mathrm{mol}$ for dihedral types $1-58$, +12.178 or $-12.178 \mathrm{kcal} / \mathrm{mol}$ for dihedral types $91,92,94$, and $95,+9.80$ or $-9.80 \mathrm{kcal} / \mathrm{mol}$ for dihedral types 93 and 96, to ensure retention of the specified stereochemistry. This effectively prevents any isomerization due to the cross-linking minimization process. After the cross-linking minimization process was completed, the potentials were returned to the values specified in Table S4.

To prevent Coulombic overlap during the cross-linking minimization, atom types 7 and 14 were given sigma values of $1.0 \AA$ and epsilon values of $0.05 \mathrm{kcal} / \mathrm{mol}$ to provide a repulsive core. After the crosslinking minimization process was completed, the sigma and epsilon parameters of these atom types returned to the values listed in Table S1.

\section{Atom Typing Descriptions}

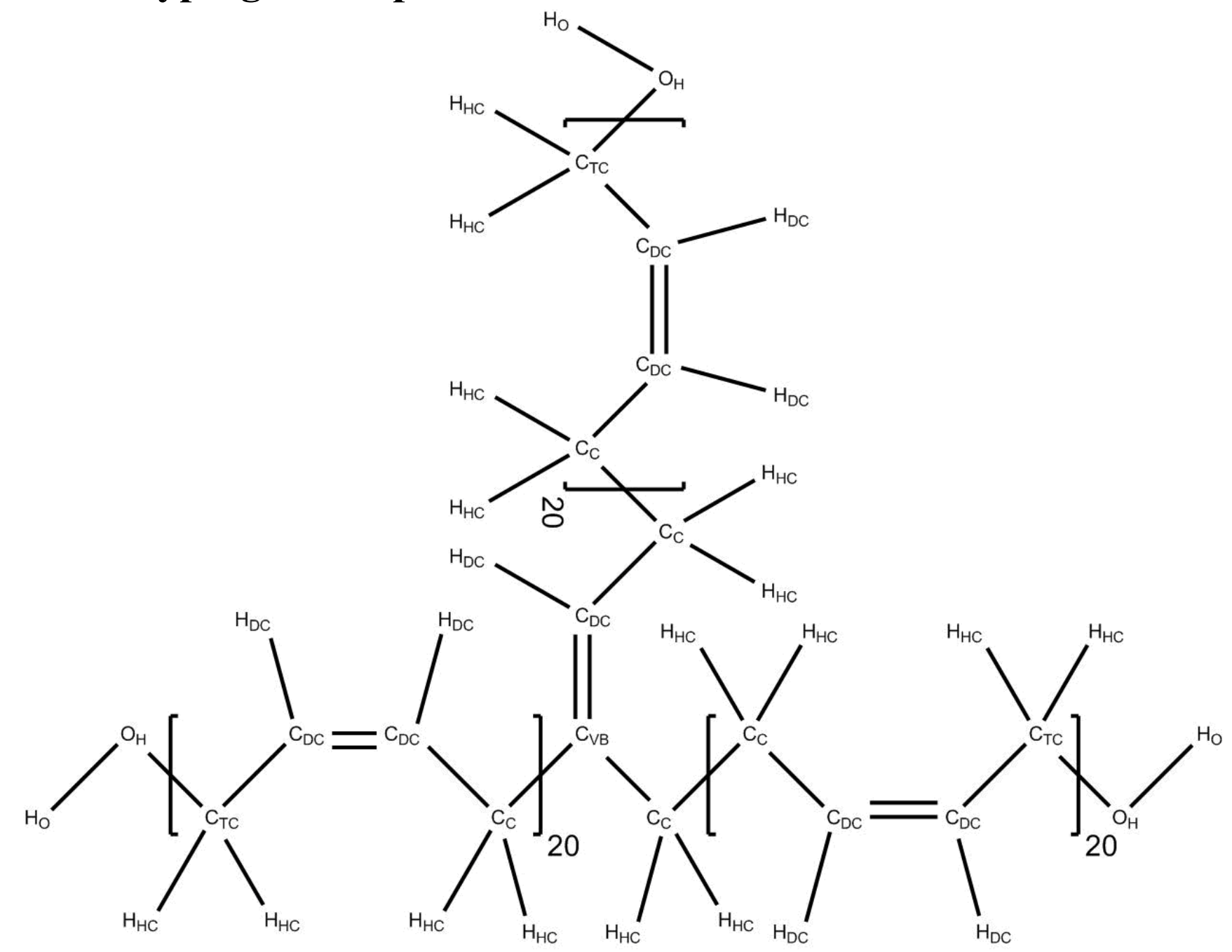


Figure S1: Atom typing for trifunctional HTPB.

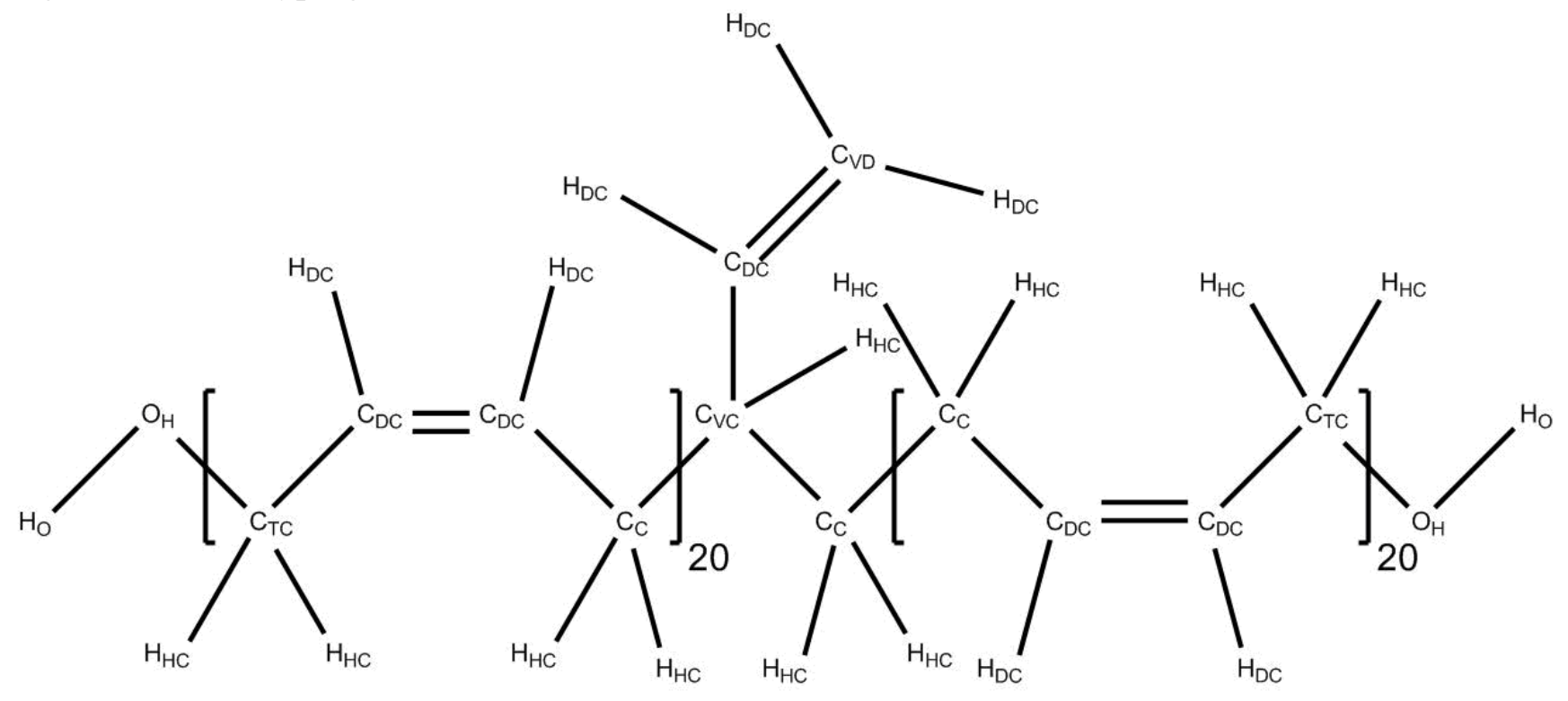

Figure S2: Atom typing for difunctional HTPB.

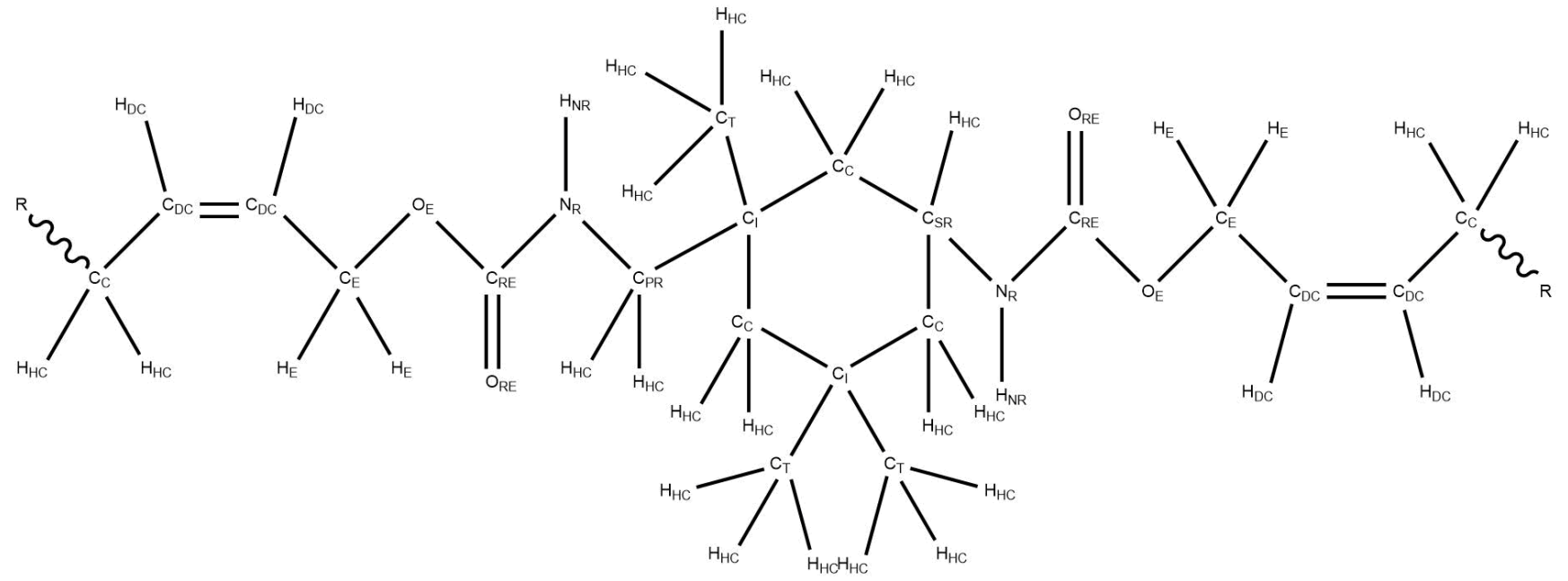

Figure S3: Atom typing for reacted HTPB-IPDI cross-link.

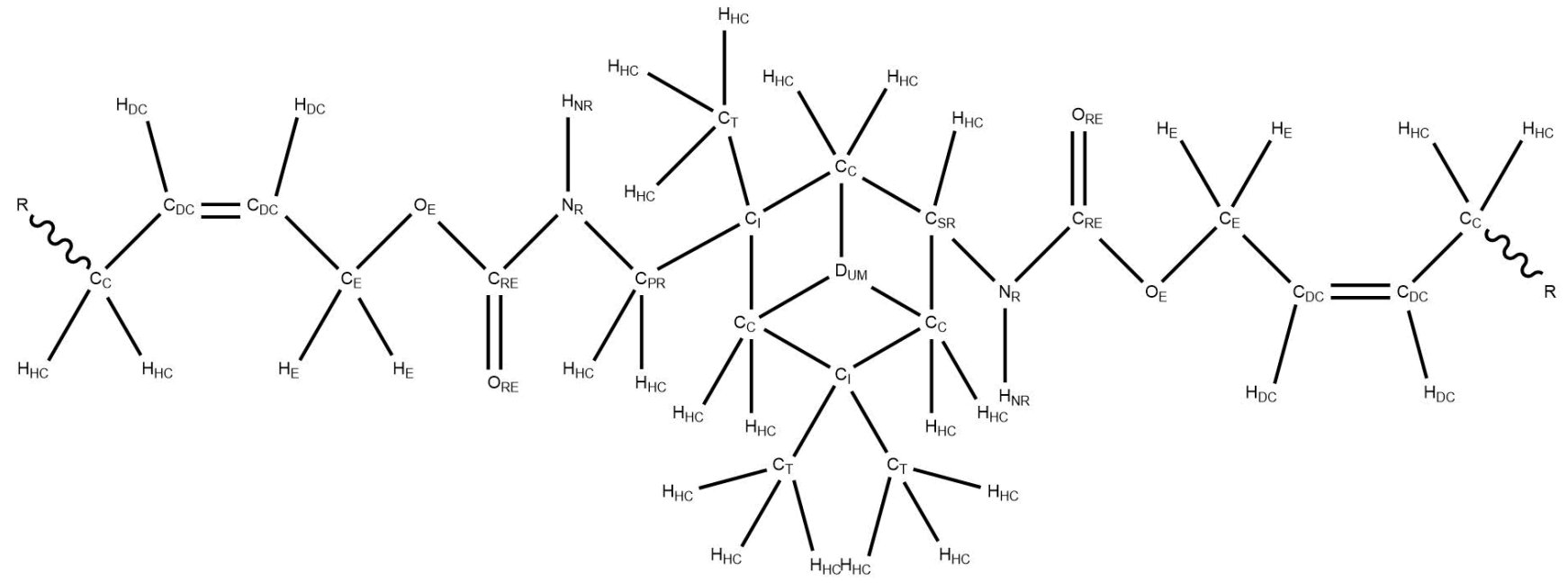

Figure S4: Atom typing for reacting HTPB-IPDI cross-link during the minimization process. 


\section{Force Field Parameters Utilized}

Table S1: Parameters for the non-bonded interactions.

\begin{tabular}{|c|c|c|c|c|c|c|}
\hline Atom Types & $\begin{array}{l}\text { LAMMPS } \\
\text { Atom Type }\end{array}$ & OPLS Type & OPLS Class & Charge & Sigma $(\AA)$ & $\begin{array}{l}\text { Epsilon } \\
(\mathrm{kcal} / \mathrm{mol})\end{array}$ \\
\hline $\mathrm{C}_{\mathrm{TC}}$ & 1 & 99 & 13 & 0.1450 & 3.5000 & 0.0660 \\
\hline $\mathrm{C}_{\mathrm{DC}}$ & 2 & 87 & 47 & -0.1150 & 3.5500 & 0.0760 \\
\hline $\mathrm{C}_{\mathrm{C}}$ & 3 & 81 & 13 & -0.1200 & 3.5000 & 0.0660 \\
\hline $\mathrm{H}_{\mathrm{HC}}$ & 4 & 85 & 46 & 0.0600 & 2.5000 & 0.0300 \\
\hline $\mathrm{H}_{\mathrm{DC}}$ & 5 & 89 & 46 & 0.1150 & 2.4200 & 0.0300 \\
\hline $\mathrm{O}_{\mathrm{H}}$ & 6 & 96 & 5 & -0.6830 & 3.1200 & 0.1700 \\
\hline $\mathrm{Ho}_{\mathrm{O}}$ & 7 & 97 & 7 & 0.4180 & 0.0000 & 0.0000 \\
\hline $\mathrm{C}_{\mathrm{VC}}$ & 8 & 82 & 13 & -0.6000 & 3.5000 & 0.0660 \\
\hline $\mathrm{C}_{\mathrm{VD}}$ & 9 & 88 & 47 & -0.2300 & 3.5500 & 0.0760 \\
\hline $\mathrm{C}_{\mathrm{VB}}$ & 10 & 86 & 47 & 0.0000 & 3.5500 & 0.0760 \\
\hline $\mathrm{C}_{\mathrm{T}}$ & 11 & 80 & 13 & -0.1800 & 3.5000 & 0.0660 \\
\hline $\mathrm{C}_{\mathrm{SR}}$ & 12 & 171 & 13 & 0.1400 & 3.5000 & 0.0660 \\
\hline $\mathrm{N}_{\mathrm{R}}$ & 13 & 180 & 24 & -0.5000 & 3.2500 & 0.1700 \\
\hline $\mathrm{H}_{\mathrm{NR}}$ & 14 & 183 & 45 & 0.3000 & 0.0000 & 0.0000 \\
\hline $\mathrm{C}_{\mathrm{RE}}$ & 15 & 406 & 3 & 0.5100 & 3.7500 & 0.1050 \\
\hline $\mathrm{O}_{\mathrm{RE}}$ & 16 & 407 & 4 & -0.4300 & 2.9600 & 0.2100 \\
\hline $\mathrm{O}_{\mathrm{E}}$ & 17 & 408 & 20 & -0.3300 & 3.0000 & 0.1700 \\
\hline $\mathrm{C}_{\mathrm{E}}$ & 18 & 431 & 13 & 0.1900 & 3.5000 & 0.0660 \\
\hline $\mathrm{H}_{\mathrm{E}}$ & 19 & 410 & 46 & 0.0300 & 2.4200 & 0.0150 \\
\hline $\mathrm{C}_{\mathrm{PR}}$ & 20 & 186 & 13 & 0.0800 & 3.5000 & 0.0660 \\
\hline $\mathrm{C}_{\mathrm{I}}$ & 21 & 84 & 13 & 0.0000 & 3.5000 & 0.0660 \\
\hline DUM & 22 & - & - & 0.0000 & 5.0000 & 0.2500 \\
\hline
\end{tabular}

Table S2: Parameters for the harmonic bond potential.

\begin{tabular}{|l|l|l|l|l|l|}
\hline Bond Types & $\begin{array}{l}\text { LAMMPS } \\
\text { Bond Type }\end{array}$ & $\begin{array}{l}\text { LAMMPS } \\
\text { Atom Types }\end{array}$ & OPLS Classes & $\begin{array}{l}\text { Nominal } \\
\text { Length }(\AA)\end{array}$ & $\begin{array}{l}\text { Force Constant } \\
(\mathrm{kcal} / \mathrm{mol})\end{array}$ \\
\hline $\mathrm{C}_{\mathrm{TC}}-\mathrm{C}_{\mathrm{DC}}$ & 1 & $1-2$ & $13-47$ & 1.5100 & 317.00 \\
\hline $\mathrm{C}_{\mathrm{TC}}-\mathrm{H}_{\mathrm{HC}}$ & 2 & $1-4$ & $13-46$ & 1.0900 & 340.00 \\
\hline $\mathrm{C}_{\mathrm{TC}}-\mathrm{O}_{\mathrm{H}}$ & 3 & $1-6$ & $13-5$ & 1.4100 & 320.00 \\
\hline $\mathrm{C}_{\mathrm{DC}}-\mathrm{C}_{\mathrm{DC}}$ & 4 & $2-2$ & $47-47$ & 1.3400 & 549.00 \\
\hline $\mathrm{C}_{\mathrm{DC}}-\mathrm{C}_{\mathrm{C}}$ & 5 & $2-3$ & $47-13$ & 1.5100 & 317.00 \\
\hline
\end{tabular}




\begin{tabular}{|l|l|l|l|l|l|}
\hline $\mathrm{C}_{\mathrm{DC}}-\mathrm{H}_{\mathrm{DC}}$ & 6 & $2-5$ & $47-46$ & 1.0800 & 340.00 \\
\hline $\mathrm{C}_{\mathrm{DC}}-\mathrm{C}_{\mathrm{VC}}$ & 7 & $2-8$ & $47-13$ & 1.5100 & 317.00 \\
\hline $\mathrm{C}_{\mathrm{DC}}-\mathrm{C}_{\mathrm{VD}}$ & 8 & $2-9$ & $47-47$ & 1.3400 & 549.00 \\
\hline $\mathrm{C}_{\mathrm{C}}-\mathrm{C}_{\mathrm{C}}$ & 9 & $3-3$ & $13-13$ & 1.5290 & 268.00 \\
\hline $\mathrm{C}_{\mathrm{C}}-\mathrm{H}_{\mathrm{HC}}$ & 10 & $3-4$ & $13-46$ & 1.0900 & 340.00 \\
\hline $\mathrm{C}_{\mathrm{C}}-\mathrm{C}_{\mathrm{VC}}$ & 11 & $3-8$ & $13-13$ & 1.5290 & 268.00 \\
\hline $\mathrm{H}_{\mathrm{HC}}-\mathrm{C}_{\mathrm{VC}}$ & 12 & $4-8$ & $13-46$ & 1.0900 & 340.00 \\
\hline $\mathrm{H}_{\mathrm{DC}}-\mathrm{C}_{\mathrm{VD}}$ & 13 & $5-9$ & $46-47$ & 1.0800 & 340.00 \\
\hline $\mathrm{O}_{\mathrm{H}}-\mathrm{H}_{\mathrm{O}}$ & 14 & $6-7$ & $5-7$ & 0.9450 & 553.00 \\
\hline $\mathrm{C}_{\mathrm{DC}}-\mathrm{C}_{\mathrm{VB}}$ & 15 & $2-10$ & $47-47$ & 1.3400 & 549.00 \\
\hline $\mathrm{C}_{\mathrm{C}}-\mathrm{C}_{\mathrm{VB}}$ & 16 & $3-10$ & $13-47$ & 1.5100 & 317.00 \\
\hline $\mathrm{C}_{\mathrm{C}}-\mathrm{C}_{\mathrm{SR}}$ & 17 & $3-12$ & $13-13$ & 1.5290 & 268.00 \\
\hline $\mathrm{C}_{\mathrm{DC}}-\mathrm{C}_{\mathrm{E}}$ & 18 & $2-18$ & $47-13$ & 1.5100 & 317.00 \\
\hline $\mathrm{C}_{\mathrm{E}}-\mathrm{H}_{\mathrm{E}}$ & 19 & $18-19$ & $13-46$ & 1.0900 & 340.00 \\
\hline $\mathrm{C}_{\mathrm{E}}-\mathrm{O}_{\mathrm{E}}$ & 20 & $18-17$ & $13-20$ & 1.4100 & 320.00 \\
\hline $\mathrm{C}_{\mathrm{PR}}-\mathrm{C}_{\mathrm{I}}$ & 21 & $20-21$ & $13-13$ & 1.5290 & 268.00 \\
\hline $\mathrm{C}_{\mathrm{PR}}-\mathrm{H}_{\mathrm{HC}}$ & 22 & $20-4$ & $13-46$ & 1.0900 & 340.00 \\
\hline $\mathrm{C}_{\mathrm{PR}}-\mathrm{N}_{\mathrm{R}}$ & 23 & $20-13$ & $13-24$ & 1.4490 & 337.00 \\
\hline $\mathrm{C}_{\mathrm{RE}}-\mathrm{N}_{\mathrm{R}}$ & 24 & $15-13$ & $3-24$ & 1.3350 & 490.00 \\
\hline $\mathrm{C}_{\mathrm{RE}}-\mathrm{O}_{\mathrm{E}}$ & 25 & $15-17$ & $3-20$ & 1.3270 & 214.00 \\
\hline $\mathrm{C}_{\mathrm{RE}}-\mathrm{O}_{\mathrm{RE}}$ & 26 & $15-16$ & $3-4$ & 1.2290 & 570.00 \\
\hline $\mathrm{C}_{\mathrm{SR}}-\mathrm{H}_{\mathrm{HC}}$ & 27 & $12-4$ & $13-46$ & 1.0900 & 340.00 \\
\hline $\mathrm{C}_{\mathrm{SR}}-\mathrm{N}_{\mathrm{R}}$ & 28 & $12-13$ & $13-24$ & 1.4490 & 337.00 \\
\hline $\mathrm{C}_{\mathrm{T}}-\mathrm{C}_{\mathrm{I}}$ & 29 & $11-21$ & $13-13$ & 1.5290 & 268.00 \\
\hline $\mathrm{C}_{\mathrm{T}}-\mathrm{H}_{\mathrm{HC}}$ & 30 & $11-4$ & $13-46$ & 1.0900 & 340.00 \\
\hline $\mathrm{H}_{\mathrm{NR}}-\mathrm{N}_{\mathrm{R}}$ & 31 & $3-22$ & $13-13$ & 1.0100 & 434.00 \\
\hline $\mathrm{C}_{\mathrm{C}}-\mathrm{C}_{\mathrm{I}}$ & 32 & $33-24$ & 1.5290 & 268.00 \\
\hline $\mathrm{C}_{\mathrm{C}}-\mathrm{D}_{\mathrm{UM}}$ & 33 & $13-13$ & 1.5290 & 268.00 \\
\hline & & & & & \\
\hline & & 13 & & \\
\hline
\end{tabular}

Table S3: Parameters for the harmonic angle potential. * = Desired type not found in native OPLS-AA, used a similar form.

\begin{tabular}{|l|l|l|l|l|l|}
\hline Angle Type & $\begin{array}{l}\text { LAMMPS } \\
\text { Angle Type }\end{array}$ & $\begin{array}{l}\text { LAMMPS Atom } \\
\text { Type }\end{array}$ & OPLS Classes & $\begin{array}{l}\text { Nominal Angle } \\
(\text { degrees })\end{array}$ & $\begin{array}{l}\text { Force Constant } \\
\text { (kcal/mol) }\end{array}$ \\
\hline $\mathrm{C}_{\mathrm{TC}-\mathrm{C}_{\mathrm{DC}}-\mathrm{C}_{\mathrm{DC}}}$ & 1 & $1-2-2$ & $13-47-47$ & 124.00 & 70.00 \\
\hline
\end{tabular}




\begin{tabular}{|c|c|c|c|c|c|}
\hline $\mathrm{C}_{\mathrm{TC}}-\mathrm{C}_{\mathrm{DC}}-\mathrm{H}_{\mathrm{DC}}$ & 2 & $1-2-5$ & $13-47-46$ & 117.00 & 35.00 \\
\hline $\mathrm{C}_{\mathrm{TC}}-\mathrm{O}_{\mathrm{H}}-\mathrm{H}_{\mathrm{O}}$ & 3 & $1-6-7$ & $13-5-7$ & 108.50 & 55.00 \\
\hline $\mathrm{C}_{\mathrm{DC}}-\mathrm{C}_{\mathrm{TC}}-\mathrm{H}_{\mathrm{HC}}$ & 4 & $2-1-4$ & $47-13-46$ & 109.50 & 35.00 \\
\hline $\mathrm{C}_{\mathrm{DC}}-\mathrm{C}_{\mathrm{TC}}-\mathrm{O}_{\mathrm{H}}$ & $5^{*}$ & $2-1-6$ & $47 *-13-5$ & 109.50 & 50.00 \\
\hline $\mathrm{C}_{\mathrm{DC}}-\mathrm{C}_{\mathrm{DC}}-\mathrm{C}_{\mathrm{C}}$ & 6 & $2-2-3$ & $47-47-13$ & 124.00 & 70.00 \\
\hline $\mathrm{C}_{\mathrm{DC}}-\mathrm{C}_{\mathrm{DC}}-\mathrm{H}_{\mathrm{DC}}$ & 7 & $2-2-5$ & $47-47-46$ & 120.00 & 35.00 \\
\hline $\mathrm{C}_{\mathrm{DC}}-\mathrm{C}_{\mathrm{C}}-\mathrm{C}_{\mathrm{C}}$ & 8 & $2-3-3$ & $47-13-13$ & 111.10 & 63.00 \\
\hline $\mathrm{C}_{\mathrm{DC}}-\mathrm{C}_{\mathrm{C}}-\mathrm{H}_{\mathrm{HC}}$ & 9 & $2-3-4$ & $47-13-46$ & 109.50 & 35.00 \\
\hline $\mathrm{C}_{\mathrm{DC}}-\mathrm{C}_{\mathrm{C}}-\mathrm{C}_{\mathrm{VC}}$ & 10 & $2-3-8$ & $47-13-13$ & 111.10 & 63.00 \\
\hline $\mathrm{C}_{\mathrm{DC}}-\mathrm{C}_{\mathrm{VC}}-\mathrm{C}_{\mathrm{C}}$ & 11 & $2-8-3$ & $47-13-13$ & 111.10 & 63.00 \\
\hline $\mathrm{C}_{\mathrm{DC}}-\mathrm{C}_{\mathrm{VC}}-\mathrm{H}_{\mathrm{HC}}$ & 12 & $2-8-4$ & $47-13-46$ & 109.50 & 35.00 \\
\hline $\mathrm{C}_{\mathrm{DC}}-\mathrm{C}_{\mathrm{VD}}-\mathrm{H}_{\mathrm{DC}}$ & 13 & $2-9-5$ & $47-47-46$ & 120.00 & 35.00 \\
\hline $\mathrm{C}_{\mathrm{C}}-\mathrm{C}_{\mathrm{DC}}-\mathrm{H}_{\mathrm{DC}}$ & 14 & $3-2-5$ & $13-47-46$ & 117.00 & 35.00 \\
\hline $\mathrm{C}_{\mathrm{C}}-\mathrm{C}_{\mathrm{C}}-\mathrm{H}_{\mathrm{HC}}$ & 15 & $3-3-4$ & $13-13-46$ & 110.70 & 37.50 \\
\hline $\mathrm{C}_{\mathrm{C}}-\mathrm{C}_{\mathrm{C}}-\mathrm{C}_{\mathrm{VC}}$ & 16 & $3-3-8$ & $13-13-13$ & 112.70 & 58.35 \\
\hline $\mathrm{C}_{\mathrm{C}}-\mathrm{C}_{\mathrm{VC}}-\mathrm{C}_{\mathrm{C}}$ & 17 & $3-8-3$ & $13-13-13$ & 112.70 & 58.35 \\
\hline $\mathrm{C}_{\mathrm{C}}-\mathrm{C}_{\mathrm{VC}}-\mathrm{H}_{\mathrm{HC}}$ & 18 & $3-8-4$ & $13-13-46$ & 110.70 & 37.50 \\
\hline $\mathrm{H}_{\mathrm{HC}}-\mathrm{C}_{\mathrm{TC}}-\mathrm{H}_{\mathrm{HC}}$ & 19 & $4-1-4$ & 46-13-46 & 107.80 & 33.00 \\
\hline $\mathrm{H}_{\mathrm{HC}}-\mathrm{C}_{\mathrm{TC}}-\mathrm{O}_{\mathrm{H}}$ & 20 & $4-1-6$ & $46-13-5$ & 109.50 & 35.00 \\
\hline $\mathrm{H}_{\mathrm{HC}}-\mathrm{C}_{\mathrm{C}}-\mathrm{H}_{\mathrm{HC}}$ & 21 & $4-3-4$ & $46-13-46$ & 107.80 & 33.00 \\
\hline $\mathrm{H}_{\mathrm{HC}}-\mathrm{C}_{\mathrm{C}}-\mathrm{C}_{\mathrm{VC}}$ & 22 & $4-3-8$ & $46-13-13$ & 110.70 & 37.50 \\
\hline $\mathrm{H}_{\mathrm{DC}}-\mathrm{C}_{\mathrm{DC}}-\mathrm{C}_{\mathrm{VC}}$ & 23 & $5-2-8$ & $46-47-13$ & 117.00 & 35.00 \\
\hline $\mathrm{H}_{\mathrm{DC}}-\mathrm{C}_{\mathrm{DC}}-\mathrm{C}_{\mathrm{VD}}$ & 24 & $5-2-9$ & $46-47-47$ & 120.00 & 35.00 \\
\hline $\mathrm{H}_{\mathrm{DC}}-\mathrm{C}_{\mathrm{VD}}-\mathrm{H}_{\mathrm{DC}}$ & 25 & $5-9-5$ & $46-47-46$ & 117.00 & 35.00 \\
\hline $\mathrm{C}_{\mathrm{VC}}-\mathrm{C}_{\mathrm{DC}}-\mathrm{C}_{\mathrm{VD}}$ & 26 & $8-2-9$ & $13-47-47$ & 124.00 & 70.00 \\
\hline $\mathrm{C}_{\mathrm{DC}}-\mathrm{C}_{\mathrm{C}}-\mathrm{C}_{\mathrm{VB}}$ & 27 & $2-3-10$ & $47-13-47$ & 112.40 & 63.00 \\
\hline $\mathrm{C}_{\mathrm{DC}}-\mathrm{C}_{\mathrm{VB}}-\mathrm{C}_{\mathrm{C}}$ & 28 & $2-10-3$ & $47-47-13$ & 124.00 & 70.00 \\
\hline $\mathrm{C}_{\mathrm{C}}-\mathrm{C}_{\mathrm{DC}}-\mathrm{C}_{\mathrm{VB}}$ & 29 & $3-2-10$ & $13-47-47$ & 124.00 & 70.00 \\
\hline $\mathrm{C}_{\mathrm{C}}-\mathrm{C}_{\mathrm{C}}-\mathrm{C}_{\mathrm{VB}}$ & 30 & $3-3-10$ & $13-13-47$ & 111.10 & 63.00 \\
\hline $\mathrm{C}_{\mathrm{C}}-\mathrm{C}_{\mathrm{VB}}-\mathrm{C}_{\mathrm{C}}$ & 31 & $3-10-3$ & $13-47-13$ & 130.00 & 70.00 \\
\hline $\mathrm{H}_{\mathrm{HC}}-\mathrm{C}_{\mathrm{C}}-\mathrm{C}_{\mathrm{VB}}$ & 32 & $4-3-10$ & $46-13-47$ & 109.50 & 35.00 \\
\hline $\mathrm{H}_{\mathrm{DC}}-\mathrm{C}_{\mathrm{DC}}-\mathrm{C}_{\mathrm{VB}}$ & 33 & $5-2-10$ & $46-47-47$ & 120.00 & 35.00 \\
\hline $\mathrm{C}_{\mathrm{C}}-\mathrm{C}_{\mathrm{SR}}-\mathrm{C}_{\mathrm{C}}$ & 34 & $3-12-3$ & $13-13-13$ & 112.70 & 58.35 \\
\hline $\mathrm{C}_{\mathrm{C}}-\mathrm{C}_{\mathrm{SR}}-\mathrm{H}_{\mathrm{HC}}$ & 35 & $3-12-4$ & $13-13-46$ & 110.70 & 37.50 \\
\hline $\mathrm{C}_{\mathrm{C}}-\mathrm{C}_{\mathrm{SR}}-\mathrm{N}_{\mathrm{R}}$ & 36 & $3-12-13$ & $13-13-24$ & 109.70 & 80.00 \\
\hline
\end{tabular}




\begin{tabular}{|c|c|c|c|c|c|}
\hline $\mathrm{C}_{\mathrm{C}}-\mathrm{C}_{\mathrm{I}}-\mathrm{C}_{\mathrm{PR}}$ & 37 & $3-21-20$ & $13-13-13$ & 112.70 & 58.35 \\
\hline $\mathrm{C}_{\mathrm{C}}-\mathrm{C}_{\mathrm{I}}-\mathrm{C}_{\mathrm{T}}$ & 38 & $3-21-11$ & $13-13-13$ & 112.70 & 58.35 \\
\hline $\mathrm{C}_{\mathrm{DC}}-\mathrm{C}_{\mathrm{DC}}-\mathrm{C}_{\mathrm{E}}$ & 39 & $2-2-18$ & $47-47-13$ & 124.00 & 70.00 \\
\hline $\mathrm{C}_{\mathrm{DC}}-\mathrm{C}_{\mathrm{E}}-\mathrm{H}_{\mathrm{E}}$ & 40 & 2-18-19 & $47-13-46$ & 109.50 & 35.00 \\
\hline $\mathrm{C}_{\mathrm{DC}}-\mathrm{C}_{\mathrm{E}}-\mathrm{O}_{\mathrm{E}}$ & $41^{*}$ & $2-18-17$ & $47^{*}-13-20$ & 109.50 & 50.00 \\
\hline $\mathrm{C}_{\mathrm{E}}-\mathrm{C}_{\mathrm{DC}}-\mathrm{H}_{\mathrm{DC}}$ & 42 & $18-2-5$ & $13-47-46$ & 117.00 & 35.00 \\
\hline $\mathrm{C}_{\mathrm{E}}-\mathrm{O}_{\mathrm{E}}-\mathrm{C}_{\mathrm{RE}}$ & 43 & $18-17-15$ & $13-20-3$ & 116.90 & 83.00 \\
\hline $\mathrm{C}_{\mathrm{PR}}-\mathrm{C}_{\mathrm{I}}-\mathrm{C}_{\mathrm{T}}$ & 44 & $20-21-11$ & $13-13-13$ & 112.70 & 58.35 \\
\hline $\mathrm{C}_{\mathrm{PR}}-\mathrm{N}_{\mathrm{R}}-\mathrm{C}_{\mathrm{RE}}$ & 45 & $20-13-15$ & $13-24-3$ & 121.90 & 50.00 \\
\hline $\mathrm{C}_{\mathrm{PR}}-\mathrm{N}_{\mathrm{R}}-\mathrm{H}_{\mathrm{NR}}$ & 46 & 20-13-14 & $13-24-45$ & 118.40 & 38.00 \\
\hline $\mathrm{C}_{\mathrm{RE}}-\mathrm{N}_{\mathrm{R}}-\mathrm{C}_{\mathrm{SR}}$ & 47 & $15-13-12$ & $3-24-13$ & 121.90 & 50.00 \\
\hline $\mathrm{C}_{\mathrm{RE}}-\mathrm{N}_{\mathrm{R}}-\mathrm{H}_{\mathrm{NR}}$ & 48 & $15-13-14$ & $3-24-45$ & 119.80 & 35.00 \\
\hline $\mathrm{C}_{\mathrm{SR}}-\mathrm{C}_{\mathrm{C}}-\mathrm{C}_{\mathrm{I}}$ & 49 & $12-3-21$ & $13-13-13$ & 112.70 & 58.35 \\
\hline $\mathrm{C}_{\mathrm{SR}}-\mathrm{C}_{\mathrm{C}}-\mathrm{H}_{\mathrm{HC}}$ & 50 & $12-3-4$ & $13-13-46$ & 110.70 & 37.50 \\
\hline $\mathrm{C}_{\mathrm{SR}}-\mathrm{N}_{\mathrm{R}}-\mathrm{H}_{\mathrm{NR}}$ & 51 & $12-13-14$ & $13-24-45$ & 118.40 & 38.00 \\
\hline $\mathrm{C}_{\mathrm{T}}-\mathrm{C}_{\mathrm{I}}-\mathrm{C}_{\mathrm{T}}$ & 52 & $11-21-11$ & $13-13-13$ & 112.70 & 58.35 \\
\hline $\mathrm{C}_{\mathrm{I}}-\mathrm{C}_{\mathrm{C}}-\mathrm{C}_{\mathrm{I}}$ & 53 & $21-3-21$ & $13-13-13$ & 112.70 & 58.35 \\
\hline $\mathrm{C}_{\mathrm{I}}-\mathrm{C}_{\mathrm{PR}}-\mathrm{H}_{\mathrm{HC}}$ & 54 & $21-20-4$ & $13-13-46$ & 110.70 & 37.50 \\
\hline $\mathrm{C}_{\mathrm{I}}-\mathrm{C}_{\mathrm{PR}}-\mathrm{N}_{\mathrm{R}}$ & 55 & $21-20-13$ & $13-13-24$ & 109.70 & 80.00 \\
\hline $\mathrm{C}_{\mathrm{I}}-\mathrm{C}_{\mathrm{T}}-\mathrm{H}_{\mathrm{HC}}$ & 56 & $21-11-4$ & $13-13-46$ & 110.70 & 37.50 \\
\hline $\mathrm{H}_{\mathrm{E}}-\mathrm{C}_{\mathrm{E}}-\mathrm{H}_{\mathrm{E}}$ & 57 & 19-18-19 & $46-13-46$ & 107.80 & 33.00 \\
\hline $\mathrm{H}_{\mathrm{E}}-\mathrm{C}_{\mathrm{E}}-\mathrm{O}_{\mathrm{E}}$ & 58 & $19-18-17$ & $46-13-20$ & 109.50 & 35.00 \\
\hline $\mathrm{H}_{\mathrm{HC}}-\mathrm{C}_{\mathrm{PR}}-\mathrm{H}_{\mathrm{HC}}$ & 59 & $4-20-4$ & $46-13-46$ & 107.80 & 33.00 \\
\hline $\mathrm{H}_{\mathrm{HC}}-\mathrm{C}_{\mathrm{PR}}-\mathrm{N}_{\mathrm{R}}$ & 60 & $4-20-13$ & $46-13-24$ & 109.50 & 35.00 \\
\hline $\mathrm{H}_{\mathrm{HC}}-\mathrm{C}_{\mathrm{SR}}-\mathrm{N}_{\mathrm{R}}$ & 61 & $4-12-13$ & $46-13-24$ & 109.50 & 35.00 \\
\hline $\mathrm{H}_{\mathrm{HC}}-\mathrm{C}_{\mathrm{T}}-\mathrm{H}_{\mathrm{HC}}$ & 62 & 4-11-4 & $46-13-46$ & 107.80 & 33.00 \\
\hline $\mathrm{N}_{\mathrm{R}}-\mathrm{C}_{\mathrm{RE}}-\mathrm{O}_{\mathrm{E}}$ & 63 & $13-15-17$ & $24-3-20$ & 111.40 & 81.00 \\
\hline $\mathrm{N}_{\mathrm{R}}-\mathrm{C}_{\mathrm{RE}}-\mathrm{O}_{\mathrm{RE}}$ & 64 & $13-15-16$ & $24-3-4$ & 122.90 & 80.00 \\
\hline $\mathrm{O}_{\mathrm{E}}-\mathrm{C}_{\mathrm{RE}}-\mathrm{O}_{\mathrm{RE}}$ & 65 & $17-15-16$ & $20-3-4$ & 123.40 & 83.00 \\
\hline $\mathrm{C}_{\mathrm{C}}-\mathrm{C}_{\mathrm{I}}-\mathrm{C}_{\mathrm{C}}$ & 66 & $3-21-3$ & $13-13-13$ & 112.70 & 58.35 \\
\hline $\mathrm{C}_{\mathrm{I}}-\mathrm{C}_{\mathrm{C}}-\mathrm{H}_{\mathrm{HC}}$ & 67 & $21-3-4$ & $13-13-46$ & 110.70 & 37.50 \\
\hline
\end{tabular}


Table S4: Parameters for the OPLS dihedral potential. * = Desired type not found in native OPLS-AA, used a similar form.

\begin{tabular}{|c|c|c|c|c|c|c|c|}
\hline Dihedral Type & $\begin{array}{l}\text { LAMMPS } \\
\text { Dihedral } \\
\text { Type }\end{array}$ & $\begin{array}{l}\text { LAMMPS Atom } \\
\text { Types }\end{array}$ & OPLS Classes & $\begin{array}{l}\mathrm{K}_{1} \\
\text { (kcal/mol) }\end{array}$ & $\begin{array}{l}\mathrm{K}_{2} \\
\text { (kcal/mol) }\end{array}$ & $\begin{array}{l}\mathrm{K}_{3} \\
\text { (kcal/mol) }\end{array}$ & $\begin{array}{l}\mathrm{K}_{4} \\
(\mathrm{kcal} / \mathrm{mol})\end{array}$ \\
\hline $\mathrm{C}_{\mathrm{TC}}-\mathrm{C}_{\mathrm{DC}}-\mathrm{C}_{\mathrm{DC}}-\mathrm{C}_{\mathrm{C}}$ & 1 & $1-2-2-3$ & $13-47-47-13$ & 0.000 & 14.000 & 0.000 & 0.000 \\
\hline $\mathrm{C}_{\mathrm{TC}}-\mathrm{C}_{\mathrm{DC}}-\mathrm{C}_{\mathrm{DC}}-\mathrm{H}_{\mathrm{DC}}$ & 2 & $1-2-2-5$ & $13-47-47-46$ & 0.000 & 14.000 & 0.000 & 0.000 \\
\hline $\mathrm{C}_{\mathrm{DC}}-\mathrm{C}_{\mathrm{TC}}-\mathrm{O}_{\mathrm{H}}-\mathrm{H}_{\mathrm{O}}$ & 3 & $2-1-6-7$ & $47-13-5-7$ & -0.900 & 0.000 & 0.000 & 0.000 \\
\hline $\mathrm{C}_{\mathrm{DC}}-\mathrm{C}_{\mathrm{DC}}-\mathrm{C}_{\mathrm{C}}-\mathrm{C}_{\mathrm{C}}$ & 4 & $2-2-3-3$ & $47-47-13-13$ & 0.346 & 0.405 & -0.904 & 0.000 \\
\hline $\mathrm{C}_{\mathrm{DC}}-\mathrm{C}_{\mathrm{DC}}-\mathrm{C}_{\mathrm{C}}-\mathrm{H}_{\mathrm{HC}}$ & 5 & $2-2-3-4$ & $47-47-13-46$ & 0.000 & 0.000 & -0.372 & 0.000 \\
\hline $\mathrm{C}_{\mathrm{DC}}-\mathrm{C}_{\mathrm{DC}}-\mathrm{C}_{\mathrm{C}}-\mathrm{C}_{\mathrm{VC}}$ & 6 & $2-2-3-8$ & $47-47-13-13$ & 0.346 & 0.405 & -0.904 & 0.000 \\
\hline $\mathrm{C}_{\mathrm{DC}}-\mathrm{C}_{\mathrm{C}}-\mathrm{C}_{\mathrm{C}}-\mathrm{C}_{\mathrm{DC}}$ & 7* & $2-3-3-2$ & $47 *-13-13-47$ & 1.300 & -0.050 & 0.200 & 0.000 \\
\hline $\mathrm{C}_{\mathrm{DC}}-\mathrm{C}_{\mathrm{C}}-\mathrm{C}_{\mathrm{C}}-\mathrm{H}_{\mathrm{HC}}$ & 8 & $2-3-3-4$ & $47-13-13-46$ & 0.000 & 0.000 & 0.366 & 0.000 \\
\hline $\mathrm{C}_{\mathrm{DC}}-\mathrm{C}_{\mathrm{C}}-\mathrm{C}_{\mathrm{C}}-\mathrm{C}_{\mathrm{VC}}$ & 9 & $2-3-3-8$ & $47-13-13-13$ & 1.300 & -0.050 & 0.200 & 0.000 \\
\hline $\mathrm{C}_{\mathrm{DC}}-\mathrm{C}_{\mathrm{C}}-\mathrm{C}_{\mathrm{VC}}-\mathrm{C}_{\mathrm{DC}}$ & $10 *$ & $2-3-8-2$ & $47 *-13-13-47$ & 1.300 & -0.050 & 0.200 & 0.000 \\
\hline $\mathrm{C}_{\mathrm{DC}}-\mathrm{C}_{\mathrm{C}}-\mathrm{C}_{\mathrm{VC}}-\mathrm{C}_{\mathrm{C}}$ & 11 & $2-3-8-3$ & $47-13-13-13$ & 1.300 & -0.050 & 0.200 & 0.000 \\
\hline $\mathrm{C}_{\mathrm{DC}}-\mathrm{C}_{\mathrm{C}}-\mathrm{C}_{\mathrm{VC}}-\mathrm{H}_{\mathrm{HC}}$ & 12 & $2-3-8-4$ & $47-13-13-46$ & 0.000 & 0.000 & 0.366 & 0.000 \\
\hline $\mathrm{C}_{\mathrm{C}}-\mathrm{C}_{\mathrm{DC}}-\mathrm{C}_{\mathrm{DC}}-\mathrm{C}_{\mathrm{C}}$ & 13 & $3-2-2-3$ & $13-47-47-13$ & 0.000 & 14.000 & 0.000 & 0.000 \\
\hline $\mathrm{C}_{\mathrm{C}}-\mathrm{C}_{\mathrm{DC}}-\mathrm{C}_{\mathrm{DC}}-\mathrm{H}_{\mathrm{DC}}$ & 14 & $3-2-2-5$ & $13-47-47-46$ & 0.000 & 14.000 & 0.000 & 0.000 \\
\hline $\mathrm{C}_{\mathrm{C}}-\mathrm{C}_{\mathrm{C}}-\mathrm{C}_{\mathrm{VC}}-\mathrm{C}_{\mathrm{DC}}$ & 15 & $3-3-8-2$ & $13-13-13-47$ & 1.300 & -0.050 & 0.200 & 0.000 \\
\hline $\mathrm{C}_{\mathrm{C}}-\mathrm{C}_{\mathrm{C}}-\mathrm{C}_{\mathrm{VC}}-\mathrm{C}_{\mathrm{C}}$ & 16 & $3-3-8-3$ & $13-13-13-13$ & 1.300 & -0.050 & 0.200 & 0.000 \\
\hline $\mathrm{C}_{\mathrm{C}}-\mathrm{C}_{\mathrm{C}}-\mathrm{C}_{\mathrm{VC}}-\mathrm{H}_{\mathrm{HC}}$ & 17 & $3-3-8-4$ & $13-13-13-46$ & 0.000 & 0.000 & 0.300 & 0.000 \\
\hline $\mathrm{H}_{\mathrm{HC}}-\mathrm{C}_{\mathrm{TC}}-\mathrm{C}_{\mathrm{DC}}-\mathrm{C}_{\mathrm{DC}}$ & 18 & $4-1-2-2$ & $46-13-47-47$ & 0.000 & 0.000 & -0.372 & 0.000 \\
\hline $\mathrm{H}_{\mathrm{HC}}-\mathrm{C}_{\mathrm{TC}}-\mathrm{C}_{\mathrm{DC}}-\mathrm{H}_{\mathrm{DC}}$ & 19 & $4-1-2-5$ & $46-13-47-46$ & 0.000 & 0.000 & 0.318 & 0.000 \\
\hline $\mathrm{H}_{\mathrm{HC}}-\mathrm{C}_{\mathrm{TC}}-\mathrm{O}_{\mathrm{H}}-\mathrm{H}_{\mathrm{O}}$ & 20 & $4-1-6-7$ & $46-13-5-7$ & 0.000 & 0.000 & 0.352 & 0.000 \\
\hline $\mathrm{H}_{\mathrm{HC}}-\mathrm{C}_{\mathrm{C}}-\mathrm{C}_{\mathrm{C}}-\mathrm{H}_{\mathrm{HC}}$ & 21 & $4-3-3-4$ & $46-13-13-46$ & 0.000 & 0.000 & 0.300 & 0.000 \\
\hline $\mathrm{H}_{\mathrm{HC}}-\mathrm{C}_{\mathrm{C}}-\mathrm{C}_{\mathrm{C}}-\mathrm{C}_{\mathrm{VC}}$ & 22 & $4-3-3-8$ & $46-13-13-13$ & 0.000 & 0.000 & 0.300 & 0.000 \\
\hline $\mathrm{H}_{\mathrm{HC}}-\mathrm{C}_{\mathrm{C}}-\mathrm{C}_{\mathrm{VC}}-\mathrm{C}_{\mathrm{DC}}$ & 23 & $4-3-8-2$ & $46-13-13-47$ & 0.000 & 0.000 & 0.366 & 0.000 \\
\hline $\mathrm{H}_{\mathrm{HC}}-\mathrm{C}_{\mathrm{C}}-\mathrm{C}_{\mathrm{VC}}-\mathrm{C}_{\mathrm{C}}$ & 24 & $4-3-8-3$ & $46-13-13-13$ & 0.000 & 0.000 & 0.300 & 0.000 \\
\hline $\mathrm{H}_{\mathrm{HC}}-\mathrm{C}_{\mathrm{C}}-\mathrm{C}_{\mathrm{VC}}-\mathrm{H}_{\mathrm{HC}}$ & 25 & $4-3-8-4$ & $46-13-13-46$ & 0.000 & 0.000 & 0.300 & 0.000 \\
\hline $\mathrm{H}_{\mathrm{DC}}-\mathrm{C}_{\mathrm{DC}}-\mathrm{C}_{\mathrm{DC}}-\mathrm{H}_{\mathrm{DC}}$ & 26 & $5-2-2-5$ & $46-47-47-46$ & 0.000 & 14.000 & 0.000 & 0.000 \\
\hline $\mathrm{H}_{\mathrm{DC}}-\mathrm{C}_{\mathrm{DC}}-\mathrm{C}_{\mathrm{C}}-\mathrm{C}_{\mathrm{C}}$ & $27 *$ & $5-2-3-3$ & $46-47-13-13^{*}$ & 0.000 & 0.000 & 0.468 & 0.000 \\
\hline $\mathrm{H}_{\mathrm{DC}}-\mathrm{C}_{\mathrm{DC}}-\mathrm{C}_{\mathrm{C}}-\mathrm{H}_{\mathrm{HC}}$ & 28 & $5-2-3-4$ & $46-47-13-46$ & 0.000 & 0.000 & 0.318 & 0.000 \\
\hline $\mathrm{H}_{\mathrm{DC}}-\mathrm{C}_{\mathrm{DC}}-\mathrm{C}_{\mathrm{C}}-\mathrm{C}_{\mathrm{VC}}$ & $29 *$ & $5-2-3-8$ & $46-47-13-13^{*}$ & 0.000 & 0.000 & 0.468 & 0.000 \\
\hline $\mathrm{H}_{\mathrm{DC}}-\mathrm{C}_{\mathrm{DC}}-\mathrm{C}_{\mathrm{VC}}-\mathrm{C}_{\mathrm{C}}$ & $30 *$ & $5-2-8-3$ & $46-47-13-13^{*}$ & 0.000 & 0.000 & 0.468 & 0.000 \\
\hline $\mathrm{H}_{\mathrm{DC}}-\mathrm{C}_{\mathrm{DC}}-\mathrm{C}_{\mathrm{VC}}-\mathrm{H}_{\mathrm{HC}}$ & 31 & $5-2-8-4$ & $46-47-13-46$ & 0.000 & 0.000 & 0.318 & 0.000 \\
\hline
\end{tabular}




\begin{tabular}{|c|c|c|c|c|c|c|c|}
\hline $\mathrm{H}_{\mathrm{DC}}-\mathrm{C}_{\mathrm{DC}}-\mathrm{C}_{\mathrm{VD}}-\mathrm{H}_{\mathrm{DC}}$ & 32 & $5-2-9-5$ & $46-47-47-46$ & 0.000 & 14.000 & 0.000 & 0.000 \\
\hline $\mathrm{O}_{\mathrm{H}}-\mathrm{C}_{\mathrm{TC}}-\mathrm{C}_{\mathrm{DC}}-\mathrm{C}_{\mathrm{DC}}$ & $33^{*}$ & $6-1-2-2$ & $5 *-13-47-47$ & 0.500 & 0.000 & 0.000 & 0.000 \\
\hline $\mathrm{O}_{\mathrm{H}}-\mathrm{C}_{\mathrm{TC}}-\mathrm{C}_{\mathrm{DC}}-\mathrm{H}_{\mathrm{DC}}$ & $34 *$ & $6-1-2-5$ & $5 *-13-47-46$ & 0.000 & 0.000 & 0.468 & 0.000 \\
\hline $\mathrm{C}_{\mathrm{VC}}-\mathrm{C}_{\mathrm{DC}}-\mathrm{C}_{\mathrm{VD}}-\mathrm{H}_{\mathrm{DC}}$ & 35 & $8-2-9-5$ & $13-47-47-46$ & 0.000 & 14.000 & 0.000 & 0.000 \\
\hline $\mathrm{C}_{\mathrm{VD}}-\mathrm{C}_{\mathrm{DC}}-\mathrm{C}_{\mathrm{VC}}-\mathrm{C}_{\mathrm{C}}$ & 36 & $9-2-8-3$ & $47-47-13-13$ & 0.346 & 0.405 & -0.904 & 0.000 \\
\hline $\mathrm{C}_{\mathrm{VD}}-\mathrm{C}_{\mathrm{DC}}-\mathrm{C}_{\mathrm{VC}}-\mathrm{H}_{\mathrm{HC}}$ & 37 & $9-2-8-4$ & $47-47-13-46$ & 0.000 & 0.000 & -0.372 & 0.000 \\
\hline $\mathrm{C}_{\mathrm{DC}}-\mathrm{C}_{\mathrm{DC}}-\mathrm{C}_{\mathrm{C}}-\mathrm{C}_{\mathrm{VB}}$ & $38 *$ & $2-2-3-10$ & $47 *-47-13-47$ & 0.000 & -8.000 & 0.000 & 0.000 \\
\hline $\mathrm{C}_{\mathrm{DC}}-\mathrm{C}_{\mathrm{C}}-\mathrm{C}_{\mathrm{C}}-\mathrm{C}_{\mathrm{VB}}$ & $39 *$ & $2-3-3-10$ & $47 *-13-13-47$ & 1.300 & -0.050 & 0.200 & 0.000 \\
\hline $\mathrm{C}_{\mathrm{DC}}-\mathrm{C}_{\mathrm{C}}-\mathrm{C}_{\mathrm{VB}}-\mathrm{C}_{\mathrm{DC}}$ & $40^{*}$ & $2-3-10-2$ & $47-13-47-47^{*}$ & 0.000 & -8.000 & 0.000 & 0.000 \\
\hline $\mathrm{C}_{\mathrm{DC}}-\mathrm{C}_{\mathrm{C}}-\mathrm{C}_{\mathrm{VB}}-\mathrm{C}_{\mathrm{C}}$ & 41 & $2-3-10-3$ & $47-13-47-13$ & 0.000 & -8.000 & 0.000 & 0.000 \\
\hline $\mathrm{C}_{\mathrm{C}}-\mathrm{C}_{\mathrm{DC}}-\mathrm{C}_{\mathrm{VB}}-\mathrm{C}_{\mathrm{C}}$ & 42 & $3-2-10-3$ & $13-47-47-13$ & 0.000 & 14.000 & 0.000 & 0.000 \\
\hline $\mathrm{C}_{\mathrm{C}}-\mathrm{C}_{\mathrm{C}}-\mathrm{C}_{\mathrm{VB}}-\mathrm{C}_{\mathrm{DC}}$ & 43 & $3-3-10-2$ & $13-13-47-47$ & 0.346 & 0.405 & -0.904 & 0.000 \\
\hline $\mathrm{C}_{\mathrm{C}}-\mathrm{C}_{\mathrm{C}}-\mathrm{C}_{\mathrm{VB}}-\mathrm{C}_{\mathrm{C}}$ & 44 & $3-3-10-3$ & $13-13-47-13$ & 2.817 & -0.169 & 0.543 & 0.000 \\
\hline $\mathrm{H}_{\mathrm{HC}}-\mathrm{C}_{\mathrm{C}}-\mathrm{C}_{\mathrm{C}}-\mathrm{C}_{\mathrm{VB}}$ & 45 & $4-3-3-10$ & $46-13-13-47$ & 0.000 & 0.000 & 0.366 & 0.000 \\
\hline $\mathrm{H}_{\mathrm{HC}}-\mathrm{C}_{\mathrm{C}}-\mathrm{C}_{\mathrm{VB}}-\mathrm{C}_{\mathrm{DC}}$ & 46 & $4-3-10-2$ & $46-13-47-47$ & 0.000 & 0.000 & -0.372 & 0.000 \\
\hline $\mathrm{H}_{\mathrm{HC}}-\mathrm{C}_{\mathrm{C}}-\mathrm{C}_{\mathrm{VB}}-\mathrm{C}_{\mathrm{C}}$ & 47 & $4-3-10-3$ & $46-13-47-13$ & 0.000 & 0.000 & 0.300 & 0.000 \\
\hline $\mathrm{H}_{\mathrm{DC}}-\mathrm{C}_{\mathrm{DC}}-\mathrm{C}_{\mathrm{C}}-\mathrm{C}_{\mathrm{VB}}$ & 48 & $5-2-3-10$ & $46-47-13-47$ & 0.000 & -8.000 & 0.000 & 0.000 \\
\hline $\mathrm{H}_{\mathrm{DC}}-\mathrm{C}_{\mathrm{DC}}-\mathrm{C}_{\mathrm{VB}}-\mathrm{C}_{\mathrm{C}}$ & 49 & $5-2-10-3$ & $46-47-47-13$ & 0.000 & 14.000 & 0.000 & 0.000 \\
\hline $\mathrm{C}_{\mathrm{VB}}-\mathrm{C}_{\mathrm{DC}}-\mathrm{C}_{\mathrm{C}}-\mathrm{C}_{\mathrm{C}}$ & 50 & $10-2-3-3$ & $47-47-13-13$ & 0.346 & 0.405 & -0.904 & 0.000 \\
\hline $\mathrm{C}_{\mathrm{VB}}-\mathrm{C}_{\mathrm{DC}}-\mathrm{C}_{\mathrm{C}}-\mathrm{H}_{\mathrm{HC}}$ & 51 & $10-2-3-4$ & $47-47-13-46$ & 0.000 & 0.000 & -0.372 & 0.000 \\
\hline $\mathrm{C}_{\mathrm{C}}-\mathrm{C}_{\mathrm{SR}}-\mathrm{N}_{\mathrm{R}}-\mathrm{C}_{\mathrm{RE}}$ & 52 & $3-12-13-15$ & $13-13-24-3$ & 0.000 & 0.462 & 0.000 & 0.000 \\
\hline $\mathrm{C}_{\mathrm{C}}-\mathrm{C}_{\mathrm{SR}}-\mathrm{N}_{\mathrm{R}}-\mathrm{H}_{\mathrm{NR}}$ & 53 & $3-12-13-14$ & $13-13-24-45$ & 0.000 & 0.000 & 0.000 & 0.000 \\
\hline $\mathrm{C}_{\mathrm{DC}}-\mathrm{C}_{\mathrm{DC}}-\mathrm{C}_{\mathrm{E}}-\mathrm{H}_{\mathrm{E}}$ & 54 & 2-2-18-19 & $47-47-13-46$ & 0.000 & 0.000 & -0.372 & 0.000 \\
\hline $\mathrm{C}_{\mathrm{DC}}-\mathrm{C}_{\mathrm{DC}}-\mathrm{C}_{\mathrm{E}}-\mathrm{O}_{\mathrm{E}}$ & $55^{*}$ & $2-2-18-17$ & $47-47-13-20 *$ & 0.500 & 0.000 & 0.000 & 0.000 \\
\hline $\mathrm{C}_{\mathrm{DC}}-\mathrm{C}_{\mathrm{E}}-\mathrm{O}_{\mathrm{E}}-\mathrm{C}_{\mathrm{RE}}$ & $56^{*}$ & $2-18-17-15$ & $47 *-13-20-3$ & -1.220 & -0.126 & 0.422 & 0.000 \\
\hline $\mathrm{C}_{\mathrm{E}}-\mathrm{C}_{\mathrm{DC}}-\mathrm{C}_{\mathrm{DC}}-\mathrm{C}_{\mathrm{C}}$ & 57 & $18-2-2-3$ & $13-47-47-13$ & 0.000 & 14.000 & 0.000 & 0.000 \\
\hline $\mathrm{C}_{\mathrm{E}}-\mathrm{C}_{\mathrm{DC}}-\mathrm{C}_{\mathrm{DC}}-\mathrm{H}_{\mathrm{DC}}$ & 58 & $18-2-2-5$ & $13-47-47-46$ & 0.000 & 14.000 & 0.000 & 0.000 \\
\hline $\mathrm{C}_{\mathrm{SR}}-\mathrm{C}_{\mathrm{C}}-\mathrm{C}_{\mathrm{I}}-\mathrm{C}_{\mathrm{C}}$ & 59 & $12-3-21-3$ & $13-13-13-13$ & 1.300 & -0.050 & 0.200 & 0.000 \\
\hline $\mathrm{C}_{\mathrm{SR}}-\mathrm{C}_{\mathrm{C}}-\mathrm{C}_{\mathrm{I}}-\mathrm{C}_{\mathrm{PR}}$ & 60 & $12-3-21-20$ & $13-13-13-13$ & 1.300 & -0.050 & 0.200 & 0.000 \\
\hline $\mathrm{C}_{\mathrm{SR}}-\mathrm{C}_{\mathrm{C}}-\mathrm{C}_{\mathrm{I}}-\mathrm{C}_{\mathrm{T}}$ & 61 & $12-3-21-11$ & $13-13-13-13$ & 1.300 & -0.050 & 0.200 & 0.000 \\
\hline $\mathrm{C}_{\mathrm{I}}-\mathrm{C}_{\mathrm{C}}-\mathrm{C}_{\mathrm{SR}}-\mathrm{C}_{\mathrm{C}}$ & 62 & $21-3-12-3$ & 13-13-13-13 & 1.300 & -0.050 & 0.200 & 0.000 \\
\hline $\mathrm{C}_{\mathrm{I}}-\mathrm{C}_{\mathrm{C}}-\mathrm{C}_{\mathrm{SR}}-\mathrm{H}_{\mathrm{HC}}$ & 63 & $21-3-12-4$ & $13-13-13-46$ & 0.000 & 0.000 & 0.300 & 0.000 \\
\hline $\mathrm{C}_{\mathrm{I}}-\mathrm{C}_{\mathrm{C}}-\mathrm{C}_{\mathrm{SR}}-\mathrm{N}_{\mathrm{R}}$ & 64 & $21-3-12-13$ & $13-13-13-24$ & 0.845 & -0.962 & 0.713 & 0.000 \\
\hline $\mathrm{C}_{\mathrm{I}}-\mathrm{C}_{\mathrm{C}}-\mathrm{C}_{\mathrm{I}}-\mathrm{C}_{\mathrm{C}}$ & 65 & $21-3-21-3$ & $13-13-13-13$ & 1.300 & -0.050 & 0.200 & 0.000 \\
\hline $\mathrm{C}_{\mathrm{I}}-\mathrm{C}_{\mathrm{C}}-\mathrm{C}_{\mathrm{I}}-\mathrm{C}_{\mathrm{PR}}$ & 66 & $21-3-21-20$ & $13-13-13-13$ & 1.300 & -0.050 & 0.200 & 0.000 \\
\hline $\mathrm{C}_{\mathrm{I}}-\mathrm{C}_{\mathrm{C}}-\mathrm{C}_{\mathrm{I}}-\mathrm{C}_{\mathrm{T}}$ & 67 & $21-3-21-11$ & $13-13-13-13$ & 1.300 & -0.050 & 0.200 & 0.000 \\
\hline
\end{tabular}




\begin{tabular}{|c|c|c|c|c|c|c|c|}
\hline $\mathrm{C}_{\mathrm{I}}-\mathrm{C}_{\mathrm{PR}}-\mathrm{N}_{\mathrm{R}}-\mathrm{C}_{\mathrm{RE}}$ & 68 & $21-20-13-15$ & $13-13-24-3$ & 0.000 & 0.462 & 0.000 & 0.000 \\
\hline $\mathrm{C}_{\mathrm{I}}-\mathrm{C}_{\mathrm{PR}}-\mathrm{N}_{\mathrm{R}}-\mathrm{H}_{\mathrm{NR}}$ & 69 & $21-20-13-14$ & $13-13-24-45$ & 0.000 & 0.000 & 0.000 & 0.000 \\
\hline $\mathrm{H}_{\mathrm{DC}}-\mathrm{C}_{\mathrm{DC}}-\mathrm{C}_{\mathrm{E}}-\mathrm{H}_{\mathrm{E}}$ & 70 & $5-2-18-19$ & $46-47-13-46$ & 0.000 & 0.000 & 0.318 & 0.000 \\
\hline $\mathrm{H}_{\mathrm{DC}}-\mathrm{C}_{\mathrm{DC}}-\mathrm{C}_{\mathrm{E}}-\mathrm{O}_{\mathrm{E}}$ & $71 *$ & $5-2-18-17$ & $46-47-13-20^{*}$ & 0.000 & 0.000 & 0.468 & 0.000 \\
\hline $\mathrm{H}_{\mathrm{E}}-\mathrm{C}_{\mathrm{E}}-\mathrm{O}_{\mathrm{E}}-\mathrm{C}_{\mathrm{RE}}$ & 72 & $19-18-17-15$ & $46-13-20-3$ & 0.000 & 0.000 & 0.198 & 0.000 \\
\hline $\mathrm{H}_{\mathrm{HC}}-\mathrm{C}_{\mathrm{C}}-\mathrm{C}_{\mathrm{SR}}-\mathrm{C}_{\mathrm{C}}$ & 73 & $4-3-12-3$ & $46-13-13-13$ & 0.000 & 0.000 & 0.300 & 0.000 \\
\hline $\mathrm{H}_{\mathrm{HC}}-\mathrm{C}_{\mathrm{C}}-\mathrm{C}_{\mathrm{SR}}-\mathrm{H}_{\mathrm{HC}}$ & 74 & $4-3-12-4$ & $46-13-13-46$ & 0.000 & 0.000 & 0.300 & 0.000 \\
\hline $\mathrm{H}_{\mathrm{HC}}-\mathrm{C}_{\mathrm{C}}-\mathrm{C}_{\mathrm{SR}}-\mathrm{N}_{\mathrm{R}}$ & 75 & $4-3-12-13$ & $46-13-13-24$ & 0.000 & 0.000 & 0.464 & 0.000 \\
\hline $\mathrm{H}_{\mathrm{HC}}-\mathrm{C}_{\mathrm{C}}-\mathrm{C}_{\mathrm{I}}-\mathrm{C}_{\mathrm{C}}$ & 76 & $4-3-21-3$ & $46-13-13-13$ & 0.000 & 0.000 & 0.300 & 0.000 \\
\hline $\mathrm{H}_{\mathrm{HC}}-\mathrm{C}_{\mathrm{C}}-\mathrm{C}_{\mathrm{I}}-\mathrm{C}_{\mathrm{PR}}$ & 77 & $4-3-21-20$ & $46-13-13-13$ & 0.000 & 0.000 & 0.300 & 0.000 \\
\hline $\mathrm{H}_{\mathrm{HC}}-\mathrm{C}_{\mathrm{C}}-\mathrm{C}_{\mathrm{I}}-\mathrm{C}_{\mathrm{T}}$ & 78 & $4-3-21-11$ & $46-13-13-13$ & 0.000 & 0.000 & 0.300 & 0.000 \\
\hline $\mathrm{H}_{\mathrm{HC}}-\mathrm{C}_{\mathrm{PR}}-\mathrm{C}_{\mathrm{I}}-\mathrm{C}_{\mathrm{C}}$ & 79 & $4-20-21-3$ & $46-13-13-13$ & 0.000 & 0.000 & 0.300 & 0.000 \\
\hline $\mathrm{H}_{\mathrm{HC}}-\mathrm{C}_{\mathrm{PR}}-\mathrm{C}_{\mathrm{I}}-\mathrm{C}_{\mathrm{T}}$ & 80 & $4-20-21-11$ & $46-13-13-13$ & 0.000 & 0.000 & 0.300 & 0.000 \\
\hline $\mathrm{H}_{\mathrm{HC}}-\mathrm{C}_{\mathrm{PR}}-\mathrm{N}_{\mathrm{R}}-\mathrm{C}_{\mathrm{RE}}$ & 81 & $4-20-13-15$ & $46-13-24-3$ & 0.000 & 0.000 & 0.000 & 0.000 \\
\hline $\mathrm{H}_{\mathrm{HC}}-\mathrm{C}_{\mathrm{PR}}-\mathrm{N}_{\mathrm{R}}-\mathrm{H}_{\mathrm{NR}}$ & 82 & $4-20-13-14$ & $46-13-24-45$ & 0.000 & 0.000 & 0.000 & 0.000 \\
\hline $\mathrm{H}_{\mathrm{HC}}-\mathrm{C}_{\mathrm{SR}}-\mathrm{N}_{\mathrm{R}}-\mathrm{C}_{\mathrm{RE}}$ & 83 & $4-12-13-15$ & $46-13-24-3$ & 0.000 & 0.000 & 0.000 & 0.000 \\
\hline $\mathrm{H}_{\mathrm{HC}}-\mathrm{C}_{\mathrm{SR}}-\mathrm{N}_{\mathrm{R}}-\mathrm{H}_{\mathrm{NR}}$ & 84 & $4-12-13-14$ & $46-13-24-45$ & 0.000 & 0.000 & 0.000 & 0.000 \\
\hline $\mathrm{H}_{\mathrm{HC}}-\mathrm{C}_{\mathrm{T}}-\mathrm{C}_{\mathrm{I}}-\mathrm{C}_{\mathrm{C}}$ & 85 & $4-11-21-3$ & $46-13-13-13$ & 0.000 & 0.000 & 0.300 & 0.000 \\
\hline $\mathrm{H}_{\mathrm{HC}}-\mathrm{C}_{\mathrm{T}}-\mathrm{C}_{\mathrm{I}}-\mathrm{C}_{\mathrm{PR}}$ & 86 & $4-11-21-20$ & $46-13-13-13$ & 0.000 & 0.000 & 0.300 & 0.000 \\
\hline $\mathrm{H}_{\mathrm{HC}}-\mathrm{C}_{\mathrm{T}}-\mathrm{C}_{\mathrm{I}}-\mathrm{C}_{\mathrm{T}}$ & 87 & $4-11-21-11$ & $46-13-13-13$ & 0.000 & 0.000 & 0.300 & 0.000 \\
\hline $\mathrm{N}_{\mathrm{R}}-\mathrm{C}_{\mathrm{PR}}-\mathrm{C}_{\mathrm{I}}-\mathrm{C}_{\mathrm{C}}$ & 88 & $13-20-21-3$ & 24-13-13-13 & 0.845 & -0.962 & 0.713 & 0.000 \\
\hline $\mathrm{N}_{\mathrm{R}}-\mathrm{C}_{\mathrm{PR}}-\mathrm{C}_{\mathrm{I}}-\mathrm{C}_{\mathrm{T}}$ & 89 & $13-20-21-11$ & $24-13-13-13$ & 0.845 & -0.962 & 0.713 & 0.000 \\
\hline $\mathrm{N}_{\mathrm{R}}-\mathrm{C}_{\mathrm{RE}}-\mathrm{O}_{\mathrm{E}}-\mathrm{C}_{\mathrm{E}}$ & 90 & $13-15-17-18$ & $24-3-20-13$ & -2.000 & 5.000 & 0.000 & 0.000 \\
\hline $\mathrm{O}_{\mathrm{E}}-\mathrm{C}_{\mathrm{RE}}-\mathrm{N}_{\mathrm{R}}-\mathrm{C}_{\mathrm{PR}}$ & 91 & $17-15-13-20$ & $20-3-24-13$ & 2.300 & 6.089 & 0.000 & 0.000 \\
\hline $\mathrm{O}_{\mathrm{E}}-\mathrm{C}_{\mathrm{RE}}-\mathrm{N}_{\mathrm{R}}-\mathrm{C}_{\mathrm{SR}}$ & 92 & $17-15-13-12$ & $20-3-24-13$ & 2.300 & 6.089 & 0.000 & 0.000 \\
\hline $\mathrm{O}_{\mathrm{E}}-\mathrm{C}_{\mathrm{RE}}-\mathrm{N}_{\mathrm{R}}-\mathrm{H}_{\mathrm{NR}}$ & 93 & $17-15-13-14$ & $20-3-24-45$ & 0.000 & 4.900 & 0.000 & 0.000 \\
\hline $\mathrm{O}_{\mathrm{RE}}-\mathrm{C}_{\mathrm{RE}}-\mathrm{N}_{\mathrm{R}}-\mathrm{C}_{\mathrm{PR}}$ & 94 & $16-15-13-20$ & $4-3-24-13$ & 0.000 & 6.089 & 0.000 & 0.000 \\
\hline $\mathrm{O}_{\mathrm{RE}}-\mathrm{C}_{\mathrm{RE}}-\mathrm{N}_{\mathrm{R}}-\mathrm{C}_{\mathrm{SR}}$ & 95 & $16-15-13-12$ & $4-3-24-13$ & 0.000 & 6.089 & 0.000 & 0.000 \\
\hline $\mathrm{O}_{\mathrm{RE}}-\mathrm{C}_{\mathrm{RE}}-\mathrm{N}_{\mathrm{R}}-\mathrm{H}_{\mathrm{NR}}$ & 96 & $16-15-13-14$ & $4-3-24-45$ & 0.000 & 4.900 & 0.000 & 0.000 \\
\hline $\mathrm{O}_{\mathrm{RE}}-\mathrm{C}_{\mathrm{RE}}-\mathrm{O}_{\mathrm{E}}-\mathrm{C}_{\mathrm{E}}$ & 97 & $16-15-17-18$ & $4-3-20-13$ & 0.000 & 5.124 & 0.000 & 0.000 \\
\hline
\end{tabular}

Table S5: Parameters for the improper dihedral potential.

\begin{tabular}{|l|l|l|l|l|l|l|}
\hline Improper Type & $\begin{array}{l}\text { LAMMPS } \\
\text { Improper } \\
\text { Type }\end{array}$ & $\begin{array}{l}\text { LAMMPS Atom } \\
\text { Types }\end{array}$ & OPLS Classes & $\begin{array}{l}\mathrm{K} \\
(\mathrm{kcal} / \mathrm{mol})\end{array}$ & $\mathrm{d}$ & $\mathrm{n}$ \\
\hline $\mathrm{C}_{\mathrm{DC}}-\mathrm{C}_{\mathrm{TC}}-\mathrm{C}_{\mathrm{DC}}-\mathrm{H}_{\mathrm{DC}}$ & 1 & $2-1-2-5$ & $47-13-47-46$ & 30.000 & -1 & 2 \\
\hline $\mathrm{C}_{\mathrm{DC}}-\mathrm{C}_{\mathrm{DC}}-\mathrm{C}_{\mathrm{C}}-\mathrm{H}_{\mathrm{DC}}$ & 2 & $2-2-3-5$ & $47-47-13-46$ & 30.000 & -1 & 2 \\
\hline
\end{tabular}




\begin{tabular}{|l|l|l|l|l|l|l|}
$\mathrm{C}_{\mathrm{VD}}-\mathrm{H}_{\mathrm{DC}}-\mathrm{H}_{\mathrm{DC}}-\mathrm{C}_{\mathrm{DC}}$ & 3 & $9-5-5-2$ & $47-46-46-47$ & 30.000 & -1 & 2 \\
\hline $\mathrm{C}_{\mathrm{DC}}-\mathrm{C}_{\mathrm{VC}}-\mathrm{C}_{\mathrm{VD}}-\mathrm{H}_{\mathrm{DC}}$ & 4 & $2-8-9-5$ & $47-13-47-46$ & 30.000 & -1 & 2 \\
\hline $\mathrm{C}_{\mathrm{VB}}-\mathrm{C}_{\mathrm{C}}-\mathrm{C}_{\mathrm{C}}-\mathrm{C}_{\mathrm{DC}}$ & 5 & $10-3-3-2$ & $47-13-13-47$ & 30.000 & -1 & 2 \\
\hline $\mathrm{C}_{\mathrm{DC}}-\mathrm{C}_{\mathrm{VB}}-\mathrm{C}_{\mathrm{C}}-\mathrm{H}_{\mathrm{DC}}$ & 6 & $2-10-3-5$ & $47-47-13-46$ & 30.000 & -1 & 2 \\
\hline $\mathrm{C}_{\mathrm{DC}}-\mathrm{C}_{\mathrm{E}}-\mathrm{C}_{\mathrm{DC}}-\mathrm{H}_{\mathrm{DC}}$ & 7 & $2-18-2-5$ & $47-13-47-46$ & 30.000 & -1 & 2 \\
\hline $\mathrm{C}_{\mathrm{RE}}-\mathrm{O}_{\mathrm{E}}-\mathrm{N}_{\mathrm{R}}-\mathrm{O}_{\mathrm{RE}}$ & 8 & $15-17-13-16$ & $3-20-24-4$ & 21.000 & -1 & 2 \\
\hline $\mathrm{N}_{\mathrm{R}}-\mathrm{C}_{\mathrm{RE}}-\mathrm{C}_{\mathrm{SR}}-\mathrm{H}_{\mathrm{NR}}$ & 9 & $13-15-12-14$ & $24-3-13-45$ & 5.000 & -1 & 2 \\
\hline $\mathrm{N}_{\mathrm{R}}-\mathrm{C}_{\mathrm{RE}}-\mathrm{C}_{\mathrm{PR}}-\mathrm{H}_{\mathrm{NR}}$ & 10 & $13-15-20-14$ & $24-3-13-45$ & 5.000 & -1 & 2 \\
\hline
\end{tabular}

\section{Simulation Results}
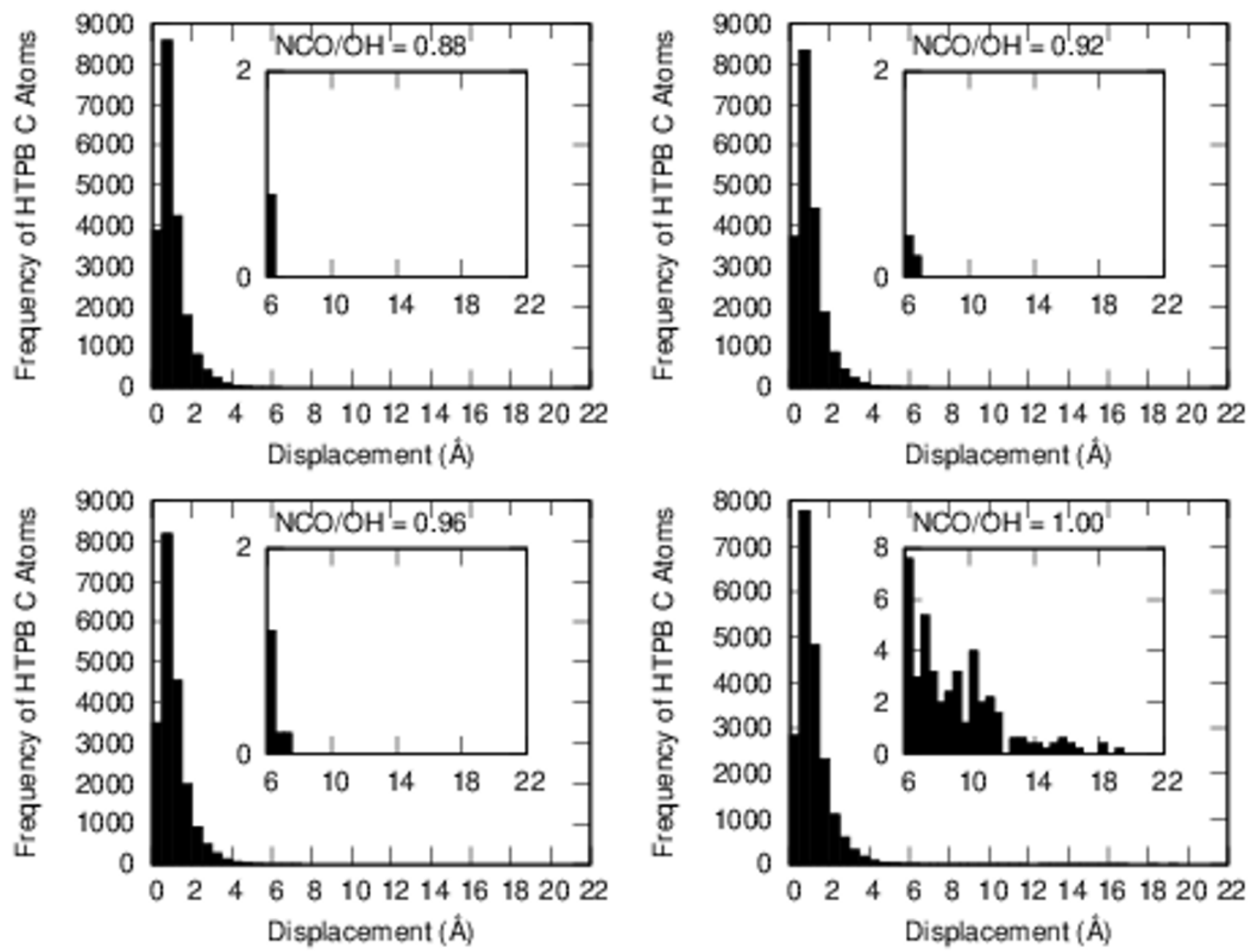

Figure S5: The relative frequency at which unreacted HTPB carbon atoms are displaced by a certain amount during the minimization procedure. Each panel corresponds to a different $\mathrm{NCO} / \mathrm{OH}$ ratio. 

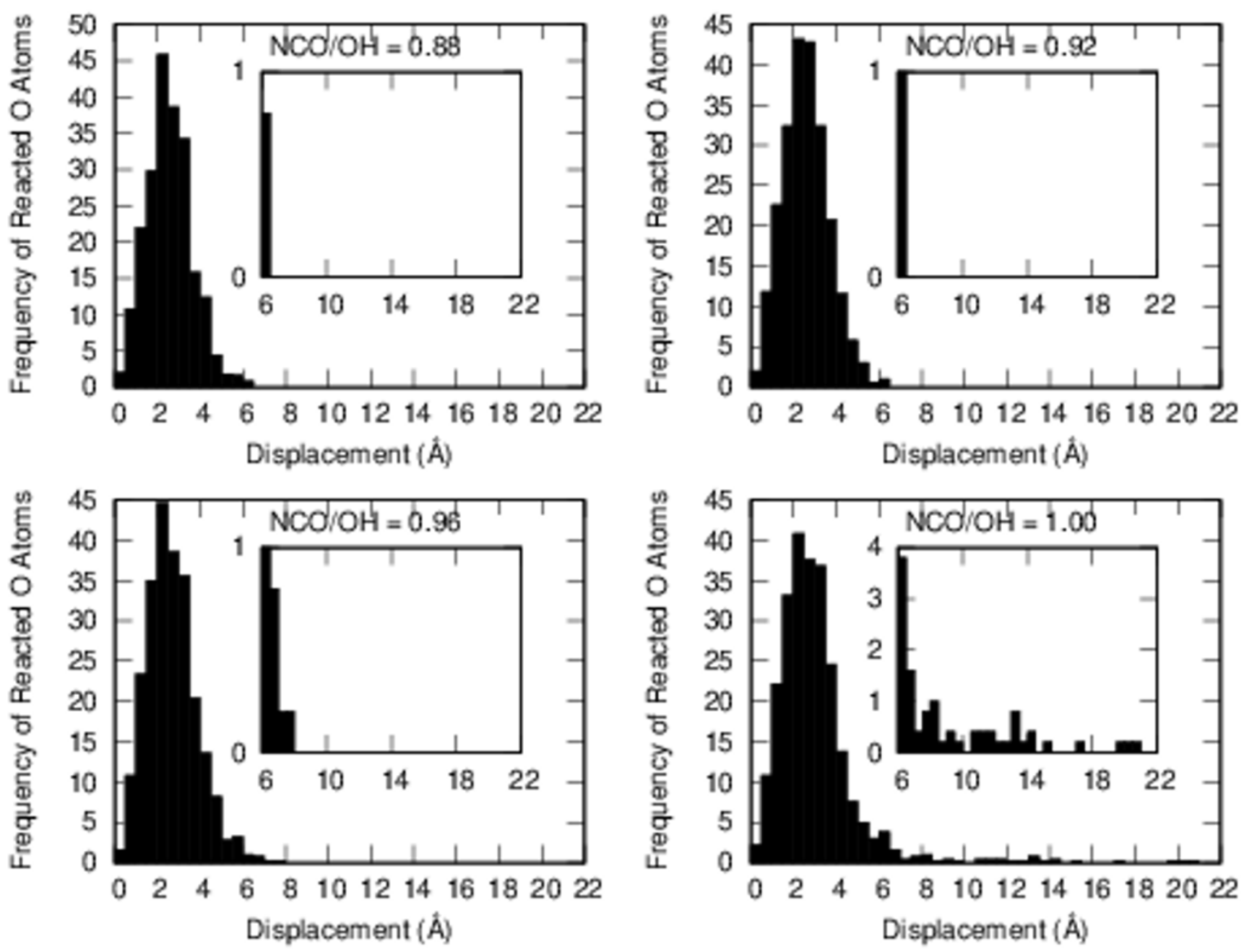

Figure S6: The relative frequency at which reacted hydroxyl oxygen atoms are displaced by a certain amount during the minimization procedure. Each panel corresponds to a different $\mathrm{NCO} / \mathrm{OH}$ ratio.

Table S6: Average density values in $\mathrm{g} / \mathrm{cm}^{3}$ for HTPB-IPDI cross-linked systems at different temperatures and $\mathrm{NCO} / \mathrm{OH}$ ratios. A sample standard deviation of approximately $0.001 \mathrm{~g} / \mathrm{cm}^{3}$ was calculated for each of the values presented in the table. The expected monotonic temperature-dependent density trend is observed. There appears to be a weak scaling relationship between the $\mathrm{NCO} / \mathrm{OH}$ ratio and the density.

\begin{tabular}{|l|l|l|l|l|}
\hline & $1.00 \mathrm{NCO} / \mathrm{OH}$ & $0.96 \mathrm{NCO} / \mathrm{OH}$ & $0.92 \mathrm{NCO} / \mathrm{OH}$ & $0.88 \mathrm{NCO} / \mathrm{OH}$ \\
\hline $233 \mathrm{~K}$ & 0.955 & 0.955 & 0.954 & 0.953 \\
\hline $253 \mathrm{~K}$ & 0.949 & 0.948 & 0.948 & 0.946 \\
\hline $273 \mathrm{~K}$ & 0.940 & 0.938 & 0.938 & 0.937 \\
\hline $293 \mathrm{~K}$ & 0.927 & 0.926 & 0.925 & 0.925 \\
\hline $313 \mathrm{~K}$ & 0.915 & 0.913 & 0.913 & 0.911 \\
\hline
\end{tabular}




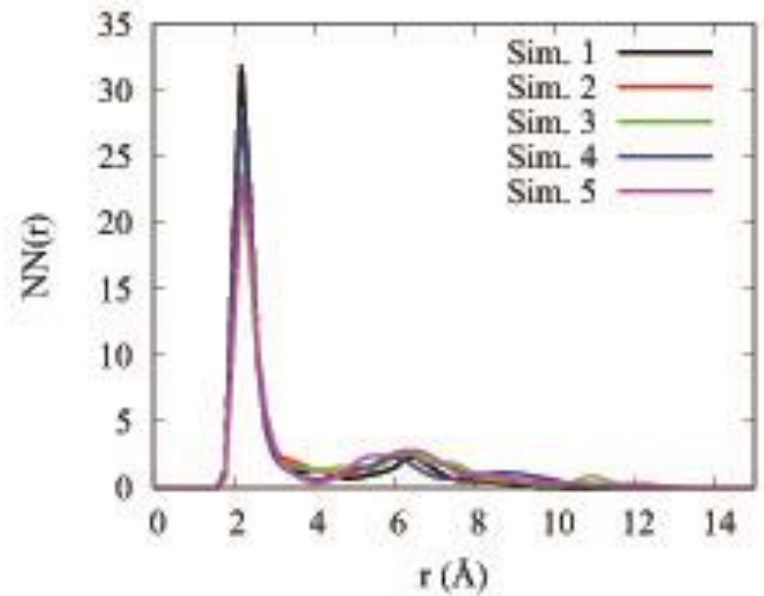

Figure S7: The relative frequency of IPDI--IPDI nearest neighbor distances. Results shown are the five systems cross-linked using a $1.00 \mathrm{NCO} / \mathrm{OH}$ ratio at $313 \sim \mathrm{K}$.

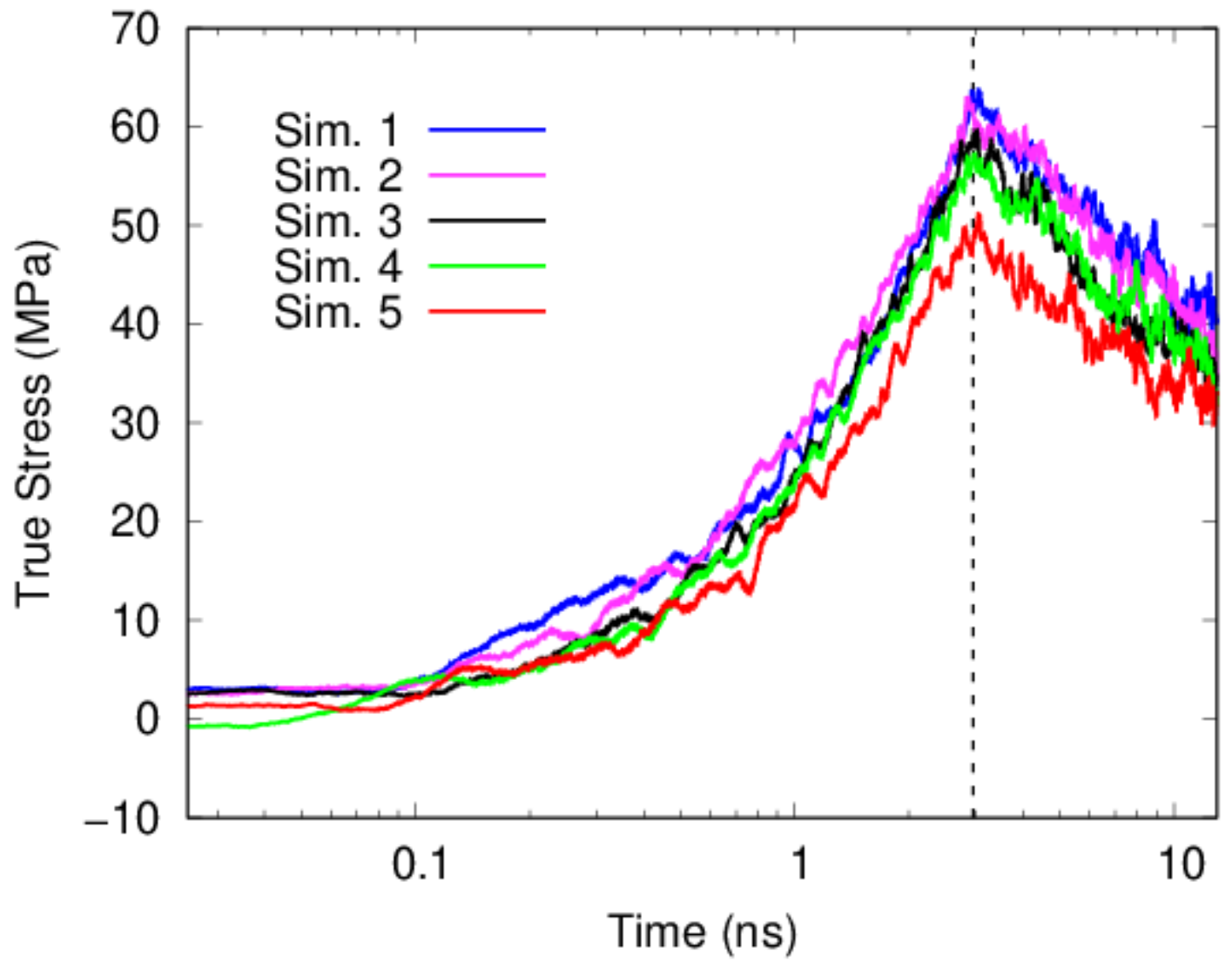

Figure S8: The true stress-time behavior for configurationally independent simulations using an $\mathrm{NCO} / \mathrm{OH}$ ratio of 1.00 and a temperature of $233 \mathrm{~K}$ from tensile deformation simulations at a true strain rate of $10^{7} \mathrm{~s}^{-1}$. The vertical dotted line denotes when the strain is held constant at $3 \%$ strain. Each plotted line is generated by averaging three simulations in which the $x$-, $y$-, or $z$-dimension is the direction of tensile deformation. The data is subsequently time averaged with a centered $50 \mathrm{ps}$ window. 


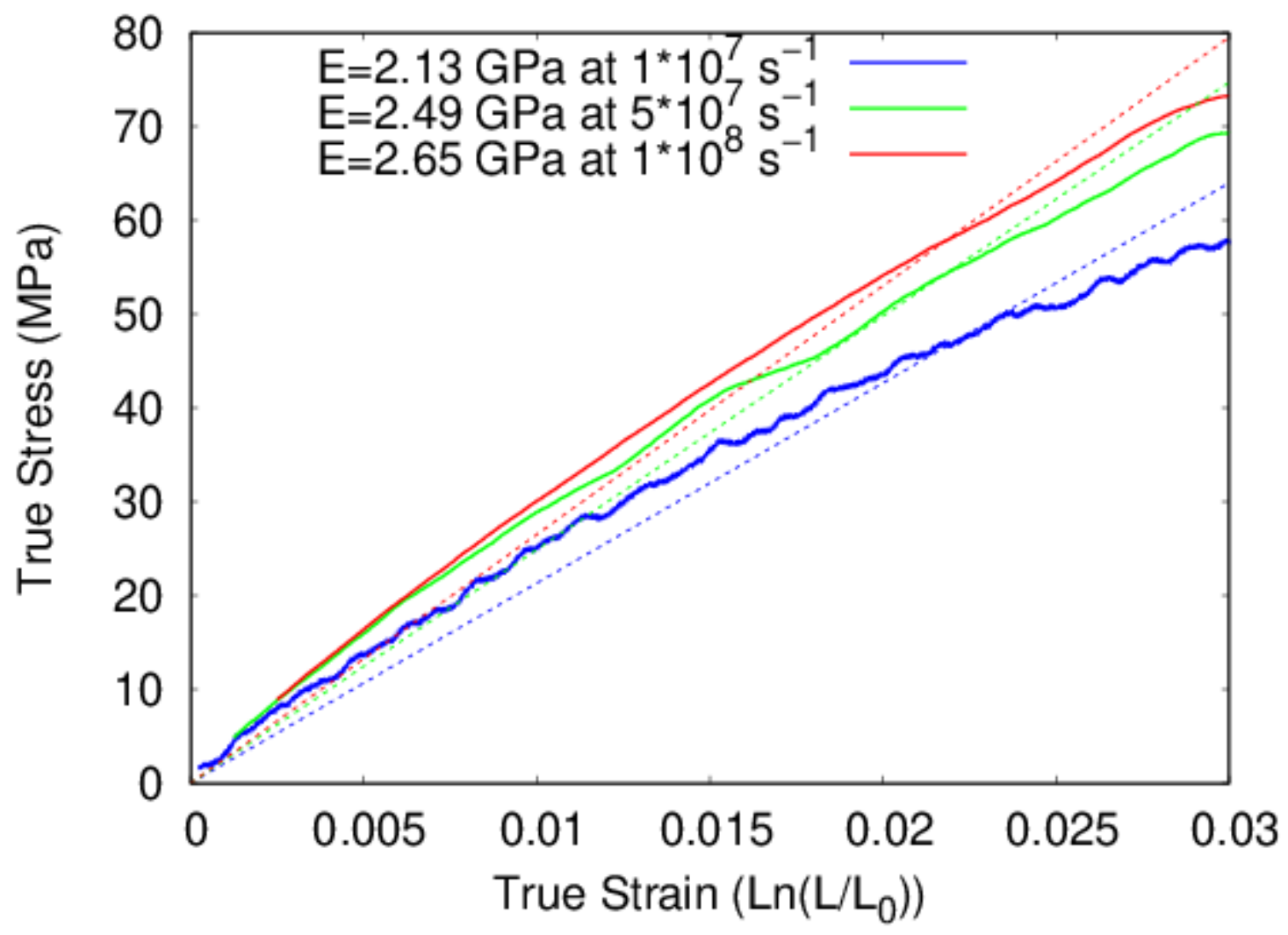

Figure S9: The true stress-strain behavior during tensile deformation at different strain rates. Linear fits to the data of each strain rate are shown by dashed lines. Young's modulus for each strain rate is shown in the legend and is calculated as the slope of a linear fit which passes through the origin. Results shown are for systems cross-linked using a $1.00 \mathrm{NCO} / \mathrm{OH}$ ratio at $233 \mathrm{~K}$. The relatively poor linear fits may indicate that the high strain rate causes non-linear stress-strain behavior, despite being in a low strain regime. As expected, faster strain rates result in higher stress for a given strain. 

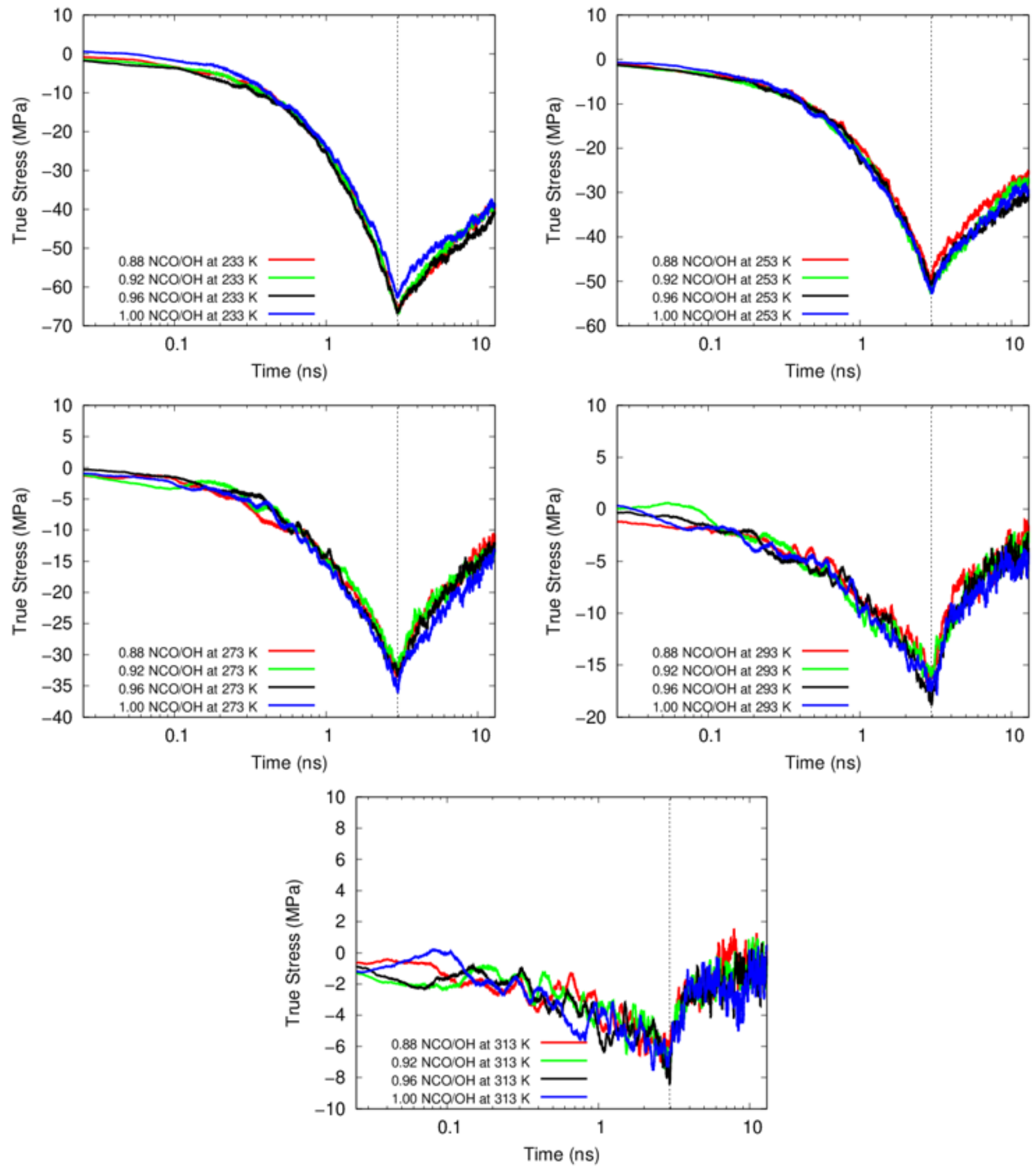

Figure S10: The true stress-time behavior at various temperatures and $\mathrm{NCO} / \mathrm{OH}$ ratios from compressive deformation simulations at a true strain rate of $10^{7} \mathrm{~s}^{-1}$. The vertical dotted line denotes when the strain is held constant at 3\% strain. Each panel is a separate temperature showing the variation in the stress behavior as a function of the $\mathrm{NCO} / \mathrm{OH}$ ratio. Each plotted line is made by averaging five configurationally independent systems deformed in three separate dimensions for a total of 15 simulations and then subsequently time averaging the data with a centered $50 \mathrm{ps}$ window. With 20 different temperature- $\mathrm{NCO} / \mathrm{OH}$ combinations, the above plots were generated using 300 simulations. Unfortunately, it appears that at these time scales the model is insensitive to the examined $\mathrm{NCO} / \mathrm{OH}$ range. 


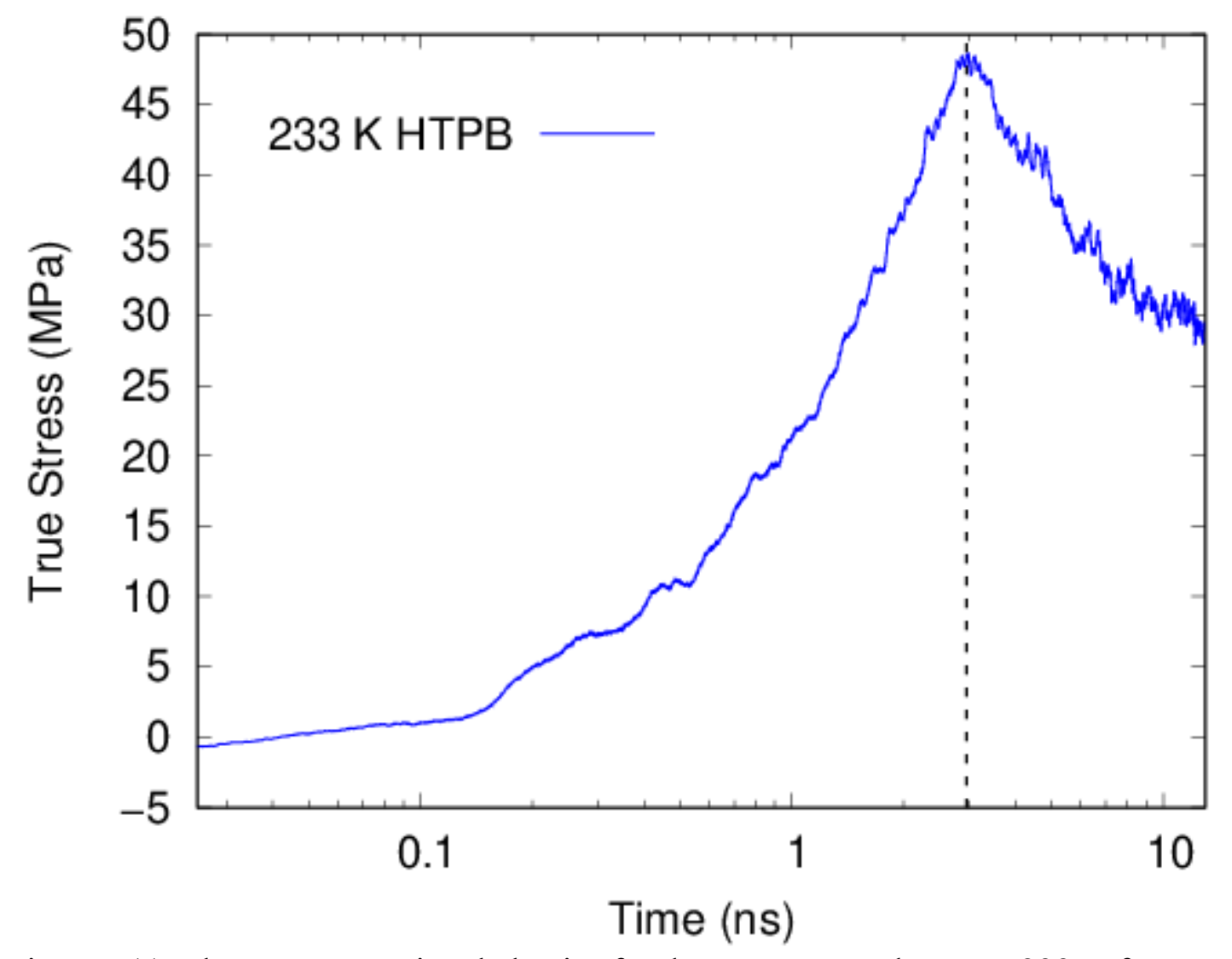

Figure S11: The true stress-time behavior for the HTPB prepolymer at $233 \mathrm{~K}$ from tensile deformation simulations at a true strain rate of $10^{7} \mathrm{~s}^{-1}$. The vertical dotted line denotes when the strain is held constant at $3 \%$ strain.

The pure HTPB deformation simulations were performed identically to the HTPB-IPDI simulations except that the pure HTPB simulations had any center-of-mass motion subtracted off from each atom and the velocities rescaled to the targeted temperature every $100 \mathrm{fs}$. This was done to prevent the 'flying ice cube' effect. Initially, simulations for pure HTPB were performed without compensating for center-of-mass drift; however, two out of the fifteen simulations experienced the flying ice cube effect. The cause was not identified. The true stress-time behavior of the thirteen out of fifteen simulations that did not experience the flying ice cube effect gave quantitatively similar results in comparison to this figure. This provides confidence that the adjustments made to the linear momentum had little to no effect on the true stress-time behavior. When comparing the peak stress between the pure HTPB system and the HTPB-IPDI $(1.00 \mathrm{NCO} / \mathrm{OH})$ system, both at $233 \mathrm{~K}$, the pure HTPB system has a peak stress that is approximately $10 \mathrm{MPa}$ lower. This indicates that there is indeed a significant quantitative difference in the stress-strain behavior that is dependent on how many IPDI crosslinks are introduced to the system. The $0.88-1.00 \mathrm{NCO} / \mathrm{OH}$ ratio was likely too narrow a range to provide for significant quantitative differences between $\mathrm{NCO} / \mathrm{OH}$ ratios when evaluating stress-strain behavior on such a short timescale. 